\title{
Commutation classes of the reduced words for the longest element of $\mathfrak{S}_{n}$
}

\author{
Gonçalo Gutierres* Ricardo Mamede* José Luis Santos* \\ University of Coimbra, CMUC, Department of Mathematics \\ Portugal \\ \{ggutc, mamede, zeluis\}@mat.uc.pt
}

Submitted: Mar 31, 2020; Accepted: May 6, 2020; Published: May 15, 2020

(C) The authors. Released under the CC BY-ND license (International 4.0).

\begin{abstract}
Using the standard Coxeter presentation for the symmetric group $\mathfrak{S}_{n}$, two reduced expressions for the same group element $w$ are said to be commutationally equivalent if one expression can be obtained from the other one by applying a finite sequence of commutations. The commutation classes can be seen as the vertices of a graph $\widehat{G}($ w), where two classes are connected by an edge if elements of those classes differ by a long braid relation. We compute the radius and diameter of the graph $\widehat{G}\left(\mathrm{w}_{\mathbf{0}}\right)$, for the longest element $\mathrm{w}_{\mathbf{0}}$ in the symmetric group $\mathfrak{S}_{n}$, and show that it is not a planar graph for $n \geqslant 6$. We also describe a family of commutation classes which contains all atoms, that is classes with one single element, and a subfamily of commutation classes whose elements are in bijection with standard Young tableaux of certain moon-polyomino shapes.
\end{abstract}

Mathematics Subject Classifications: 05A05, 05A19, 05C12

\section{Introduction}

Given a positive integer $n \geqslant 2$, let $\mathfrak{S}_{n+1}$ denote the symmetric group on the alphabet $[n+1]:=\{1,2, \ldots, n+1\}$, with composition of permutations performed from left to right. We represent a permutation $\mathrm{w} \in \mathfrak{S}_{n+1}$ as the word $\mathrm{w}=\mathrm{w}(1) \mathrm{w}(2) \cdots \mathrm{w}(n+1)$ in one-line notation.

*This work was partially supported by the Centre for Mathematics of the University of Coimbra UIDB/00324/2020, funded by the Portuguese Government through FCT/MCTES. We are thankful to Diogo Soares for the implementation of the software that allowed the visualization of the graph $\widehat{G}\left(\mathrm{w}_{0}\right)$. 
The symmetric group $\mathfrak{S}_{n+1}$ is generated by the simple reflections $\left\{\mathbf{s}_{1}, \mathbf{s}_{2}, \ldots, \mathbf{s}_{n}\right\}$, where $\mathbf{s}_{i}$ is the transposition $(i i+1)$. These reflections satisfy the Coxeter relations:

$$
\begin{aligned}
\mathrm{s}_{i}^{2} & =1, \quad \text { for all } i, \\
\mathrm{~s}_{i} \mathrm{~s}_{j} & =\mathrm{s}_{j} \mathrm{~s}_{i}, \quad \text { for }|i-j|>1, \text { and } \\
\mathrm{s}_{i} \mathrm{~s}_{i+1} \mathrm{~s}_{i} & =\mathrm{s}_{i+1} \mathrm{~s}_{i} \mathrm{~s}_{i+1}, \text { for all } i \leqslant n-1 .
\end{aligned}
$$

The relations (1) are known as commutations or short braid relations, and the relations (2) are called long braid relations. Since $\left\{\mathbf{s}_{1}, \mathbf{s}_{2}, \ldots, \mathbf{s}_{n}\right\}$ generates $\mathfrak{S}_{n+1}$, any permutation $\mathbf{w} \in \mathfrak{S}_{n+1}$ can be written as a product of adjacent transpositions $\mathbf{w}=\mathbf{s}_{i_{1}} \mathbf{s}_{i_{2}} \cdots \mathbf{s}_{i_{\ell}}$.

Consider $\mathbf{w} \in \mathfrak{S}_{n+1}$ written as a product $\mathbf{w}=\mathbf{s}_{i_{1}} \mathbf{s}_{i_{2}} \cdots \mathrm{s}_{i_{\ell}}$ where $i_{j} \in[n]$ and $\ell$ is minimal. The length of $\mathrm{w}$ is $\ell(\mathrm{w}):=\ell$ and the product $\mathrm{s}_{i_{1}} \mathrm{~s}_{i_{2}} \cdots \mathrm{s}_{i_{\ell(\mathrm{w})}}$ is a reduced decomposition for $\mathrm{w}$. The string of subscripts $w=i_{1} i_{2} \cdots i_{\ell}$ is a reduced word for $\mathrm{w}$. The content of $w$ is the sequence $\operatorname{cont}(w)=\left(c_{1}, \ldots, c_{n}\right)$, where each $c_{i}$ is the number of occurrences of the letter $i$ in $w$. A consecutive substring of $w$ is called a factor, and a word obtained by deleting some of the letters of $w$ is a subword of $w$. If $I \subseteq[n]$, then $w_{\mid I}$ is the subword of $w$ formed by the letters in $I$.

We will use sans-serif typeface for permutations, in order to distinguish them from reduced words. Reduced decompositions and reduced words are in bijection with each other, and the terms "commutation" and "long braid relation" have natural interpretations in the context of reduced words. The set of all reduced words of $w$ is denoted by $R(\mathrm{w})$.

The length $\ell(w)$ of a permutation $w$ can be computed by counting inversions (see [4]):

$$
\ell(\mathrm{w})=\mid\{(i, j): i<j \text { and } \mathrm{w}(i)>\mathrm{w}(j)\} \mid .
$$

As an immediate consequence of $(3)$, we have $\ell(w)=\ell\left(w^{-1}\right)$ for any permutation $w \in$ $\mathfrak{S}_{n+1}$.

The symmetric group $\mathfrak{S}_{n+1}$ has a unique longest element $\mathbf{w}_{\mathbf{0}} \in \mathfrak{S}_{n+1}$ with length $\ell\left(\mathrm{w}_{\mathbf{0}}\right)=\left(\begin{array}{c}n+1 \\ 2\end{array}\right)$. In one-line notation, $\mathrm{w}_{\mathbf{0}}$ is the permutation $(n+1) n \cdots 321$. The word $w_{0}:=1(21)(321) \cdots(n \cdots 21)$ is a reduced word for $\mathbf{w}_{\mathbf{0}}$. When the order of the symmetric group is not clear from the context, we will often use the notation $\mathbf{w}_{\mathbf{0}}^{n}$ for $\mathbf{w}_{\mathbf{0}} \in \mathfrak{S}_{n+1}$ to emphasize the underlying set $\{1, \ldots, n\}$ of $\mathbf{w}_{0}$.

We define a relation $\sim$ on the set $R\left(\mathrm{w}_{\mathbf{0}}\right)$ of all reduced words for $\mathrm{w}_{\mathbf{0}}$ by setting $s \sim t$ if and only if $s$ and $t$ differ by a sequence of commutations. This is an equivalence relation and the classes it defines are the commutation classes of $\mathrm{w}_{\mathbf{0}}$, denoted by $C\left(\mathrm{w}_{\mathbf{0}}\right)$. The commutation class of a word $w \in R\left(\mathbf{w}_{\mathbf{0}}\right)$ is denoted by $[w]$. Although two words in the same commutative class have the same content, this property is not sufficient to characterize the class. The next lemma gives a characterization of the words in a commutative class (see [2]).

Lemma 1. Let $v$ and $w$ be words over the alphabet $[n]$. Then, $w \sim v$ if and only if for each integer $i \in[n-1]$, we have $w_{\mid\{i, i+1\}}=v_{\mid\{i, i+1\}}$. 
Proof. If $w \sim v$, then $w$ can be obtained from $v$ by a sequence of commutative relations that do not change the relative positions of the letters $i$ and $i+1$, and so we must have $w_{\mid\{i, i+1\}}=v_{\mid\{i, i+1\}}$ for all $i \in[n-1]$. Reciprocally, if $w_{\mid\{i, i+1\}}=v_{\mid\{i, i+1\}}$ for all $i \in[n-1]$, then the relative positions of the letters $i$ and $i+1$ is the same for both words $v$ and $w$, for all $i \in[n-1]$, and thus $v$ and $w$ can differ only by the positions of non consecutive integers $i$ and $j$ with $|i-j|>1$, that can be transposed using commutation relations. It follows that $w \sim v$.

We write $v \underset{\mathrm{s}}{\sim} w($ resp. $v \underset{\mathrm{L}}{\sim} w)$ when $v$ and $w$ differ by a single short (resp., long) braid relation, and $[v] \sim[w]$ when those classes differ by a long braid relation, i.e. if there are $v^{\prime} \in[v]$ and $w^{\prime} \in[w]$ such that $v^{\prime} \sim w^{\prime}$.

Definition 2. The graph $\widehat{G}\left(w_{0}\right)$ of commutation classes of $\mathbf{w}_{\mathbf{0}} \in \mathfrak{S}_{n+1}$ has vertex set $C\left(\mathbf{w}_{\mathbf{0}}\right)$, and an edge between classes $[u]$ and $[w]$ when $[u] \sim[w]$.

The graph $\widehat{G}\left(\mathrm{w}_{\mathbf{0}}\right)$ can be seen as a quotient graph of $G\left(\mathrm{w}_{\mathbf{0}}\right)$, the graph whose vertex set is the set $R\left(\mathrm{w}_{\mathbf{0}}\right)$ of all reduced words of $\mathrm{w}_{\mathbf{0}}$, and where two reduced words are connected by an edge if they are related by a single short or long braid relation $[9,10]$.

The distance $d([u],[w])$ between commutation classes $[u]$ and $[w]$ in $\widehat{G}\left(\mathrm{w}_{\mathbf{0}}\right)$ is the length of a shortest path joining $[u]$ and $[w]$. The eccentricity of $[w]$ is the distance to a farthest commutative class from $[w]$. The radius and diameter of $\widehat{G}\left(\mathrm{w}_{\mathbf{0}}\right)$ are the minimum and maximum eccentricities, respectively. Figure 1 depicts the graph $\widehat{G}\left(\mathbf{w}_{\mathbf{0}}\right)$ for $\mathfrak{S}_{4}$, which has radius and diameter equal to 4 . We will prove that for $n \geqslant 2$, both the radius and diameter of $\widehat{G}\left(\mathrm{w}_{\mathbf{0}}^{n}\right)$ are given by the binomial $\left(\begin{array}{c}n+1 \\ 3\end{array}\right)$.

Some of the only work on this topic was obtained by S. Elnitsky [7], who proved that $\widehat{G}\left(w_{0}\right)$ is a connected and bipartite graph, by establishing a bijection between reduced words and rhombic tilings of a certain polygon $[6,13]$. In this paper we study various properties of $\widehat{G}\left(\mathrm{w}_{\mathbf{0}}\right)$, namely we compute its radius and diameter, and show that it is not planar for $n>5$. We note that the diameter of the graph $G\left(\mathrm{w}_{\mathbf{0}}\right)$ was obtained by V. Reiner and Y. Roichman in [9]. We also describe a family of commutation classes which contains all atoms, that is classes with one single word, and a subfamily of commutation classes whose elements are in bijection with standard Young tableaux of certain moon-polyomino shapes.

The remainder of this paper is organized as follows. In Section 2, we compute the radius and diameter of $\widehat{G}\left(\mathrm{w}_{\mathbf{0}}^{n}\right)$ using two statistics on reduced words, and prove that this graph is not planar for $n>4$. After some preliminary discussions, in Section 3 we describe the set of two side ordered words, a certain family of reduced words which contains as particular cases all atoms and alternating words, which are those reduced words $w$ for which each subword $w_{\mid\{a, b\}}$ is of the form $(a b)^{k}$ or $(a b)^{k} a$ for some integer $k$, whenever $|a-b|=1$. The characterization of the atoms in $\widehat{G}\left(\mathrm{w}_{\mathbf{0}}^{n}\right)$ is achieved in Subsection 3.1, and in Subsection 3.2 the notion of an alternating words is introduced and its commutation classes are characterized. Finally, in Subsection 3.3 we give an interpretation of the 
commutation classes of alternating words as standard fillings of certain moon-polyomino Young tableaux. We prove that the cardinality of each commutation class of an alternating word is larger than the cardinality of any class connected to it by a single edge, and present a conjecture with the identification of the commutation classes with maximum cardinality amongst all classes in $R\left(\mathrm{w}_{\mathbf{0}}^{n}\right)$.

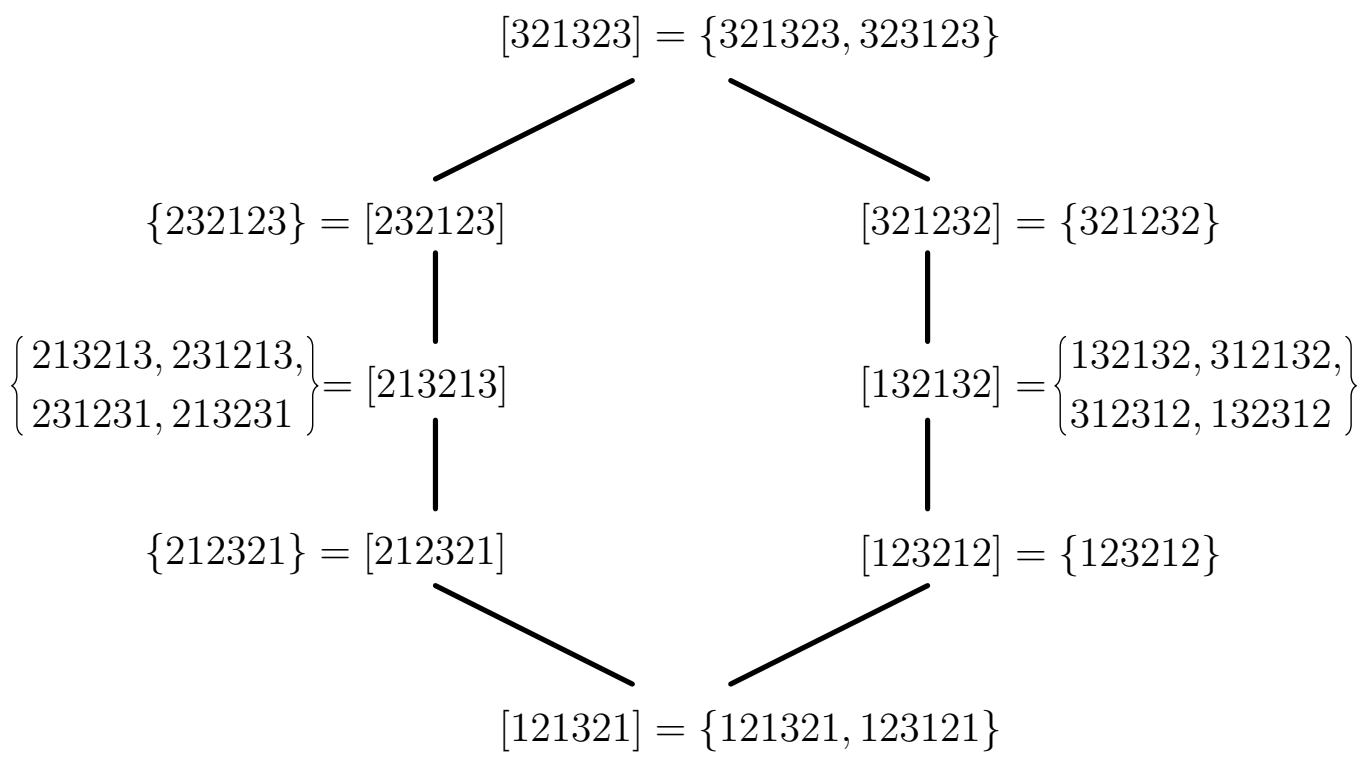

Figure 1: The graph $\widehat{G}\left(\mathrm{w}_{\mathbf{0}}^{3}\right)$.

\section{Radius, diameter and planarity}

Given a reduced word $w=i_{1} i_{2} \cdots i_{\ell}$ for $\mathbf{w}_{\mathbf{0}} \in \mathfrak{S}_{n+1}$, define the complement $w^{\bullet}$ and reverse $w^{\mathrm{R}}$ words of $w$ as

$$
w^{\bullet}:=\left(n-i_{1}+1\right)\left(n-i_{2}+1\right) \cdots\left(n-i_{\ell}+1\right) \quad \text { and } \quad w^{\mathrm{R}}:=i_{\ell} \cdots i_{2} i_{1} .
$$

The complement and reverse operations define involutive maps $R\left(\mathrm{w}_{\mathbf{0}}\right) \rightarrow R\left(\mathrm{w}_{\mathbf{0}}\right)$ that commute with each other, $\left(w^{\bullet}\right)^{\mathrm{R}}=\left(w^{\mathrm{R}}\right)^{\bullet}$, and $\operatorname{cont}(w)=\operatorname{cont}\left(w^{\mathrm{R}}\right)$.

Example 3. In $\mathfrak{S}_{4}, w_{0}=121321, w_{0}^{\bullet}=323123, w_{0}^{\mathrm{R}}=123121$ and $w_{0}^{\bullet \mathrm{R}}=w^{\mathrm{R} \bullet}=321323$.

Definition 4. Given a reduced word $w=i_{1} i_{2} \cdots i_{\ell} \in R\left(\mathrm{w}_{\mathbf{0}}\right)$, let $S(w)$ be the sum of all $\ell$ letters of $w$, that is

$$
S(w)=\sum_{j=1}^{\ell} i_{j}=\sum_{j=1}^{n} j \cdot c_{j},
$$

where $\operatorname{cont}(w)=\left(c_{1}, \ldots, c_{n}\right)$. 
The number $S(w)$ is invariant for any word in the equivalence class of $[w]$, and thus defines a map $C\left(\mathrm{w}_{\mathbf{0}}\right) \rightarrow \mathbb{N}$. For instance, we have

$$
S\left(w_{0}\right)=S\left(w_{0}^{\mathrm{R}}\right)=\sum_{j=1}^{n} j(n-j+1)=\frac{n(n+1)(n+2)}{6}
$$

and

$$
S\left(w_{0}^{\bullet}\right)=S\left(w_{0}^{\bullet R}\right)=\sum_{j=1}^{n} j^{2}=\frac{n(n+1)(2 n+1)}{6} .
$$

The next lemma follows directly from the definition of a long braid relation.

Lemma 5. If $v \underset{\mathrm{L}}{\sim}$, then $|S(v)-S(w)|=1$.

Proposition 6. Let $v \in R\left(w_{0}\right)$. If $[v] \neq\left[w_{0}\right]$ then there is $u \in R\left(w_{\mathbf{0}}\right)$ such that $[u] \underset{\mathrm{L}}{\sim}[v]$ and $S(u)<S(v)$.

Proof. We prove the contrapositive assertion. Let $v \in R\left(\mathbf{w}_{\mathbf{0}}\right)$, and assume that for any class $[u] \sim[v]$ we have $S(u) \geqslant S(v)$. Then, by Lemma $5, S(u)=S(v)+1$. This implies that a factor $i(i-1) i$ cannot appear in any word of the class $[v]$, for any $i=2, \ldots, n$. Since between two letters $n$ in a reduced word for $w_{0}$ there must be a letter $n-1$, then a word in the class $[v]$ can only have one letter $n$.

Between two consecutive letters $n-1$ in a reduced word for $w_{\mathbf{0}}$, there must be a letter $n$ or a letter $n-2$. Since we have established that a word in the class $[v]$ cannot have a factor $i(i-1) i$, for any $i$, it follows that there must exist a word in $[v]$ having the factor $(n-1) n(n-1)$ or $(n-1) n(n-2)(n-1)$. In any case, in between two consecutive letters $n-1$ it has to appear the letter $n$. This implies that any word in $[v]$ has at most two letters $n-1$.

Repeating this argument, we conclude that any word in $[v]$ has at most $i$ letters $n-i+1$, for $i=1, \ldots, n$, and that between two consecutive letters $i$, there is exactly one letter $i+1$, for all $i$. On the other hand, being reduced, the length of the words in $[v]$ is $\left(\begin{array}{c}n+1 \\ 2\end{array}\right)$. Thus, any word in $[v]$ has exactly $i$ letters $n-i+1$, and $v_{\mid\{i-1, i\}}=(i-1 i)^{n-i+1}(i-1)=\left(w_{0}\right)_{\mid\{i-1, i\}}$ for $i=1, \ldots, n$. By Lemma 1, it follows that $v \sim w_{0}$.

The previous result shows that $S\left(w_{0}\right)$ is the minimum value for the map $S$, and that $S(w)=S\left(w_{0}\right)$ if and only if $w \in\left[w_{0}\right]$. Moreover, an analogous argument shows that $S\left(w_{0}^{\bullet}\right)$ is the maximum value for the map $S$ and $S(w)=S\left(w_{0}^{\bullet}\right)$ if and only if $w \in\left[w_{0}^{\bullet}\right]$. That is, $S\left(w_{0}\right) \leqslant S(w) \leqslant S\left(w_{0}^{\bullet}\right)$ for any $w \in R\left(\mathbf{w}_{\mathbf{0}}\right)$. Since $S\left(\mathbf{w}_{\mathbf{0}}\right)=S\left(\mathbf{w}_{\mathbf{0}}^{\mathrm{R}}\right)$ and $S\left(\mathbf{w}_{\mathbf{0}}^{\bullet}\right)=S\left(\mathbf{w}_{\mathbf{0}}^{\bullet \mathrm{R}}\right)$, we can also conclude that $w_{0} \sim w_{0}^{\mathrm{R}}$ and $w_{0}^{\bullet} \sim w_{0}^{\bullet \mathrm{R}}$.

It also follows from Proposition 6 that for any commutation class $[w]$, distinct from $\left[w_{0}\right]$, there is a path from $[w]$ to a class with a smaller $S$-value, which means that there is a path joining $[w]$ and $\left[w_{0}\right]$. Thus, we recover the following result from S. Elnitsky [7], which is also a consequence of Matsumoto's Theorem [8]. This result also follows from the fact that $G(\mathbf{w})$ is connected for any $\mathbf{w} \in \mathfrak{S}_{n+1}[9]$. 
Corollary 7. The graph $\widehat{G}\left(w_{\mathbf{0}}\right)$ is connected.

Additionally, by Lemma 5 it follows that $\widehat{G}\left(\mathrm{w}_{0}\right)$ is a bipartite graph, with the partition of $C\left(\mathrm{w}_{0}\right)$ given by the parity of the $S$-values of its vertices. This result also holds for the graph $G(\mathrm{w})$, for any $\mathrm{w} \in \mathfrak{S}_{n+1}$ (see [3]).

Corollary 8. The graph $\widehat{G}\left(w_{0}\right)$ is bipartite.

The map $S$ may also be used to compute the diameter of $\widehat{G}\left(\mathrm{w}_{\mathbf{0}}\right)$.

Theorem 9. The diameter of $\widehat{G}\left(w_{\mathbf{0}}\right)$ is $S\left(w_{0}^{\bullet}\right)-S\left(w_{0}\right)=\left(\begin{array}{c}n+1 \\ 3\end{array}\right)$.

Proof. By Proposition 6, there is $w \in R\left(\mathrm{w}_{\mathbf{0}}\right)$ such that $[w] \sim\left[w_{0}^{\bullet}\right]$, and moreover $S(w)=$ $S\left(w_{0}^{\bullet}\right)-1$ by Lemma 5 . Repeating this process $S\left(w_{0}^{\bullet}\right)-S\left(w_{0}\right)$ times, we arrive at the class $\left[w_{0}\right]$, proving that

$$
d\left(\left[w_{0}\right],\left[w_{0}^{\bullet}\right]\right)=S\left(w_{0}^{\bullet}\right)-S\left(w_{0}\right)=\frac{(n-1) n(n+1)}{6}=\left(\begin{array}{c}
n+1 \\
3
\end{array}\right) .
$$

Similarly, we can see that for any class $[w]$ we have $d\left(\left[w_{0}\right],[w]\right)=S(w)-S\left(w_{0}\right)$ and $d\left([w],\left[w_{0}^{\bullet}\right]\right)=S\left(w_{0}^{\bullet}\right)-S(w)$.

To prove that the diameter is $\left(\begin{array}{c}n+1 \\ 3\end{array}\right)$, it remains to show that this number is the maximal distance between any two classes in the graph. Consider two commutation classes $[w]$ and $\left[w^{\prime}\right]$. Since

$$
d\left(\left[w_{0}\right],[w]\right)+d\left([w],\left[w_{0}^{\bullet}\right]\right)+d\left(\left[w_{0}\right],\left[w^{\prime}\right]\right)+d\left(\left[w^{\prime}\right],\left[w_{0}^{\bullet}\right]\right)=2\left(S\left(w_{0}^{\bullet}\right)-S\left(w_{0}\right)\right),
$$

using the triangle inequality, we conclude that

$$
\begin{aligned}
d\left([w],\left[w^{\prime}\right]\right) & \leqslant \min \left\{d\left(\left[w_{0}\right],[w]\right)+d\left(\left[w_{0}\right],\left[w^{\prime}\right]\right), d\left([w],\left[w_{0}^{\bullet}\right]\right)+d\left(\left[w^{\prime}\right],\left[w_{0}^{\bullet}\right]\right)\right\} \\
& \leqslant \frac{1}{2}\left(d\left(\left[w_{0}\right],[w]\right)+d\left(\left[w_{0}\right],\left[w^{\prime}\right]\right)+d\left([w],\left[w_{0}^{\bullet}\right]\right)+d\left(\left[w^{\prime}\right],\left[w_{0}^{\bullet}\right]\right)\right) \\
& =S\left(w_{0}^{\bullet}\right)-S\left(w_{0}\right),
\end{aligned}
$$

proving that the distance between any two commutation classes $[w]$ and $\left[w^{\prime}\right]$ is at most $S\left(w_{0}^{\bullet}\right)-S\left(w_{0}\right)$. It follows that the maximum eccentricity of a commutation class in the graph $\widehat{G}\left(\mathrm{w}_{\mathbf{0}}\right)$ is $S\left(w_{0}^{\bullet}\right)-S\left(w_{0}\right)$.

We will now prove that the eccentricity of any commutation class is, in fact, $\left(\begin{array}{c}n+1 \\ 3\end{array}\right)$ and therefore prove that the radius of $R\left(\mathrm{w}_{\mathbf{0}}\right)$ is equal to the diameter. To this end, we will define an auxiliary function $T$. A generator $s_{i}$ acts on a permutation $x_{1} x_{2} \cdots x_{n+1}$ of $[n+1]$ by transposing the integers $x_{i}$ and $x_{i+1}$. In particular, a reduced word $w=i_{1} i_{2} \cdots i_{\ell}$ for $\mathbf{w}_{\mathbf{0}}$ transforms the identity $12 \cdots(n+1)$, which has no inversions, into $\mathbf{w}_{\mathbf{0}}=(n+1) \cdots 21$, where each pair of integers $(i, j)$ with $1 \leqslant i<j \leqslant n+1$ is an inversion, by the successive action of the generators $\mathrm{s}_{i_{1}}, \mathrm{~s}_{i_{2}}, \ldots, \mathrm{s}_{i_{\ell}}$. 
Let $T_{n}$ be the set of all triples $(a, b, c) \in[n+1]^{3}$ such that $a<b<c$. For any $w \in R\left(\mathrm{w}_{\mathbf{0}}\right)$ and any $(a, b, c) \in T_{n}$ define $T(w, a b c)=1$ if, by the action of the generators of $w$ on the identity, the inversion of the pair $(a, b)$ occurs before the inversion of $(b, c)$; and define $T(w, a b c)=-1$ otherwise. The number $T(w, a b c)$ can be easily read off of the line diagram of a permutation. The line diagram of $\mathbf{w}=\mathrm{s}_{i_{1}} \mathbf{s}_{i_{2}} \cdots \mathrm{s}_{i_{\ell}}$ is the array $[n+1] \times[\ell]$ in the Cartesian coordinates, which describes the trajectories of the numbers $1,2, \ldots, n+1$ as they are arranged into the permutation $w$ by the successive simple transpositions $s_{i_{j}}$. Note that since $w$ is a reduced word for $\mathbf{w}_{\mathbf{0}}$, any two integers $a<b$ in $[n+1]$ will invert positions in the line diagram of $w$, thus showing that $T$ is well defined.

Figure 2 shows the line diagram of the word $w=212321 \in \widehat{G}\left(\mathbf{w}_{\mathbf{0}}^{4}\right)$, and it follows that $T(w, 123)=-1$ and $T(w, a b c)=1$ for all remaining triples $(a, b, c)$, with $a<b<c$.

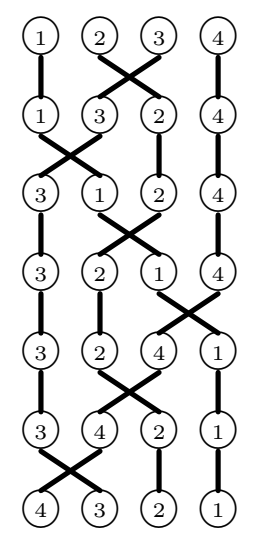

Figure 2: Line diagram of the word 212321.

Lemma 10. Two reduced words $w, w^{\prime} \in R\left(w_{0}\right)$ are in the same commutation class if and only if $T(w, a b c)=T\left(w^{\prime}, a b c\right)$ for all triples $(a, b, c) \in T_{n}$.

Proof. The operator $T$ is invariant for words in the same commutation class, since any possible change of order of the generators is done between generators acting on disjoint pairs of numbers.

Reciprocally, suppose $T(w, a b c)=T\left(w^{\prime}, a b c\right)$ and let $i$ be the leftmost letter of $w$ such that $w=w_{1} i w_{2}$ and $w^{\prime}=w_{1} j w_{2}^{\prime}$ with $i \neq j$. Then, $w^{\prime}=w_{1} u i v$, where $w_{1} u$ has no letter $i$. If $w_{1} u$ has a letter $i-1$, then the first occurrence of $i-1$ inverts the order of the integers in a pair $(a, b)$ with $a<b=i$. On the other hand, the generator $i$ in the word $w$, inverts the order of the integers in a pair $(b, c)$ where $b=i<c=i+1$. Thus, $T(w, a b c)=-1$ and $T\left(w^{\prime}, a b c\right)=1$. It follows that $w_{1} u$ cannot have the letter $i-1$, and similar reasoning shows that it cannot have $i+1$. By commutation relations we can write $w^{\prime} \sim w^{\prime \prime}$, where $w^{\prime \prime}=w_{1} i w_{2}^{\prime \prime}$. Repeating this argument for the successive words obtained from $w^{\prime}$ until the resulting word is $w$, we conclude that $w \sim w^{\prime}$.

In view of the result above, we will write $T([w], a b c)$ to represent the common number $T(v, a b c)$ for all $v \in[w]$. 
Lemma 11. If $[w] \underset{\mathrm{L}}{\sim}\left[w^{\prime}\right]$, then $T(w, a b c)=T\left(w^{\prime}, a b c\right)$ for all triples in $T_{n}$, except for one.

Proof. Without loss of generality, let $w=u i(i+1) i v \in R\left(\mathrm{w}_{\mathbf{0}}\right)$ and $w^{\prime}=u(i+1) i(i+1) v$, where $u$ and $v$ are, respectively, the initial and final factors of $w$ and $w^{\prime}$. If $a, b, c$ are such that $\mathrm{u}(a)=i, \mathrm{u}(b)=i+1$ and $\mathrm{u}(c)=i+2$, where $\mathrm{u}$ is the permutation corresponding to $u$, then $a<b<c$ since otherwise $w$ would not be reduced. Moreover, the permutation corresponding to the left factor $u i(i+1) i$ is equal to the one corresponding to $u(i+$ 1) $i(i+1)$. The transposition corresponding to the generator $i$, applied to the permutation $\mathrm{u}$, inverts the integers $a$ and $b$, while the transposition corresponding to the $i+1$ applied to $\mathrm{u}$ inverts the integers $b$ and $c$. Therefore, $T(w, a b c)=-T\left(w^{\prime}, a b c\right)$. Finally, since the permutation corresponding to $i(i+1) i=(i+1) i(i+1)$ only acts over $a, b$ and $c$, for any other triple $x<y<z$, we have $T(w, x y z)=T\left(w^{\prime}, x y z\right)$.

Proposition 12. For any $w \in R\left(w_{0}\right)$ and any triple $(a, b, c) \in T_{n}$, we have

(a) $T([w], a b c)=-T\left(\left[w^{\bullet}\right],(n+2-c)(n+2-b)(n+2-a)\right)$;

(b) $T([w], a b c)=T\left(\left[w^{R}\right],(n+2-c)(n+2-b)(n+2-a)\right)$;

(c) $T([w], a b c)=-T\left(\left[w^{\bullet R}\right], a b c\right)$.

Proof. (a) The line diagram of $w^{\bullet}$ corresponds to the horizontal reflection of the line diagram of $w$. Since the word $w^{\bullet}$ consists in replacing each generator $i$, in the word $w$, by the generator $n+1-i$, the inversion of the integers $a$ and $b$ is achieved by the action of $w$ in the same order that the inversion of the integers $n+2-a$ and $n+2-b$ is achieved by $w^{\bullet}$. And so, if the action of $w$ inverts the integers $a$ and $b$ before it inverts $b$ and $c$, then the action of $w^{\bullet}$, inverts the integers $n+2-a$ and $n+2-b$ before it inverts $n+2-b$ and $n+2-c$. Thus the result follows.

(b) Note that the line diagram of $w^{R}$ corresponds to the 180 degrees rotation of the line diagram of $w$, and the image of $a$ by $\mathrm{w}_{\mathbf{0}}$ is $n+2-a$. Reading the word $w$ backwards, any inversion of the integers $a$ and $b$ corresponds to a inversion of the integers $n+2-a$ and $n+2-b$ in the reverse order. Thus, if $a$ and $b$ are inverted before $b$ and $c$ by the action of $w$, then $n+2-b$ and $n+2-c$ are inverted before $n+2-b$ and $n+2-a$ are inverted by the action of $w^{\mathrm{R}}$. It follows that $T([w], a b c)=T\left(\left[w^{\mathrm{R}}\right], n+2-c, n+2-b, n+2-a\right)$.

(c) Follows from the previous two cases, noticing that the line diagram of $w^{\bullet R}$ corresponds to the vertical reflection of the line diagram of $w$.

Corollary 13. For any $w \in R\left(w_{0}\right)$,

$$
d\left([w],\left[w^{\bullet R}\right]\right)=\left(\begin{array}{c}
n+1 \\
3
\end{array}\right) .
$$

Proof. By Theorem 9, the distance between any two words is at most $\left(\begin{array}{c}n+1 \\ 3\end{array}\right)$. By Proposition 12, for any triple $(a, b, c), T([w], a b c)=-T\left(\left[w^{\bullet \mathrm{R}}\right], a b c\right)$ and, by Lemma 11, this means that there are necessary at least $\left|T_{n}\right|$ long relations in a path linking $[w]$ and $\left[w^{\bullet \mathbf{R}}\right]$. Thus $d\left([w],\left[w^{\bullet R}\right]\right) \geqslant\left|T_{n}\right|=\left(\begin{array}{c}n+1 \\ 3\end{array}\right)$, which concludes the proof. 
The following result is a consequence of the previous corollary and Theorem 9 .

Theorem 14. The eccentricity of any class $[w]$ is $\left(\begin{array}{c}n+1 \\ 3\end{array}\right)$, and therefore the radius of $\widehat{G}\left(w_{\mathbf{0}}\right)$ is $\left(\begin{array}{c}n+1 \\ 3\end{array}\right)$.

As can be seen in Figures 1 and 3, the graphs $\widehat{G}\left(\mathrm{w}_{0}^{n}\right)$ for $n \leqslant 4$ are planar graphs. In Figure 3, the vertices $O$ and $J$ correspond to the commutation classes of $w_{0}$ and $w_{0}^{\bullet R}$, and the vertices in each dashed circle have the same $S$-value, with one unit of increment (resp., decrement) for each circle starting from $S\left(w_{0}\right)=20$ (resp., $S\left(w_{0}^{\bullet \mathrm{R}}\right)=30$ ) in the center. Moreover, the vertices on the two external circles have the same $S$-value. For instance, the $S$-value of vertices $A_{1}$ and $A_{2}$ is 21 , and the $S$-values of $B_{1}$ and $B_{2}$ is 22 . The vertices $E_{1}$ and $E_{2}$, in the two external circles, have the same $S$-value equal to 25 , and the $S$-value of vertex $F$ is 26 .

We prove next that for $n>4$ the graph $\widehat{G}\left(\mathrm{w}_{\mathbf{0}}\right)$ is not planar, using Wagner's Theorem [5]. An edge contraction of an edge $e=\{u, v\}$ in a graph is the graph obtained by combining the vertices $u$ and $v$ into a single vertex, which is adjacent to every vertex that was adjacent to $u$ and $v$ in the original graph. A graph minor of a graph is a new graph obtained by deleting vertices, deleting edges, and/or contracting edges of the original graph. Wagner's Theorem states that a graph is planar if and only if it does not contain $K_{5}$ or $K_{3,3}$ as a graph minor.

Lemma 15. Given a fixed integer $n \geqslant 2$, the graph $\widehat{G}\left(w_{0}^{n-1}\right)$ is a subgraph of $\widehat{G}\left(w_{0}^{n}\right)$.

Proof. Notice that for any word $w$ in $\widehat{G}\left(\mathrm{w}_{\mathbf{0}}^{n-1}\right)$, the word $w^{\prime}=w \cdot w_{n}^{-}$is a word in $\widehat{G}\left(\mathrm{w}_{\mathbf{0}}^{n}\right)$, where we set $w_{n}^{-}=n(n-1) \cdots 21$. The subgraph of $\widehat{G}\left(\mathrm{w}_{0}^{n}\right)$ formed by the classes of the words $w \cdot w_{n}^{-}$, with $w \in R\left(\mathrm{w}_{0}^{n-1}\right)$, is isomorphic to $\widehat{G}\left(\mathrm{w}_{0}^{n-1}\right)$.

For example, the graph $\widehat{G}\left(\mathrm{w}_{\mathbf{0}}^{3}\right)$ can be seen as the subgraph of $\widehat{G}\left(\mathrm{w}_{\mathbf{0}}^{4}\right)$ formed by the classes denoted by $O, A_{1}, A_{2}, B_{1}, B_{2}, C_{1}, C_{2}, D$ in Figure 3, where $O=$ [1213214321], $A_{1}=$ [2123214321], $A_{2}=$ [1232124321], $B_{1}=$ [2132134321], $B_{2}=$ [1321324321], $C_{1}=$ [2321234321], $C_{2}=[3212324321]$ and $D=[3213234321]$.

Theorem 16. For $n>4$ the graph $\widehat{G}\left(w_{0}^{n}\right)$ is not planar.

Proof. Using Lemma 15 , it is enough to prove that $\widehat{G}\left(\mathrm{w}_{\mathbf{0}}^{5}\right)$ is not planar. We will do so by proving that it has $K_{3,3}$ as minor.

The minor of $\widehat{G}\left(\mathrm{w}_{\mathbf{0}}^{5}\right)$ having as vertices the sets:

$A=\{[132132432154321]\}$,

$B=\{[123214321354321]\}$,

$C=\{[123212432543212]\}$,

$D=\{[123212432154321]\}$,

$E=\{[132134321354321],[132134323543212],[123214323543212]\}$,

$F=\{[123214321543214],[132134321543214],[321234321543214],[321234325432124]$, [321234323543212], [321232432543212], [132132432543212]\},

where $E$ and $F$ are the edge contractions of their vertices, is isomorphic to $K_{3,3}$, since 


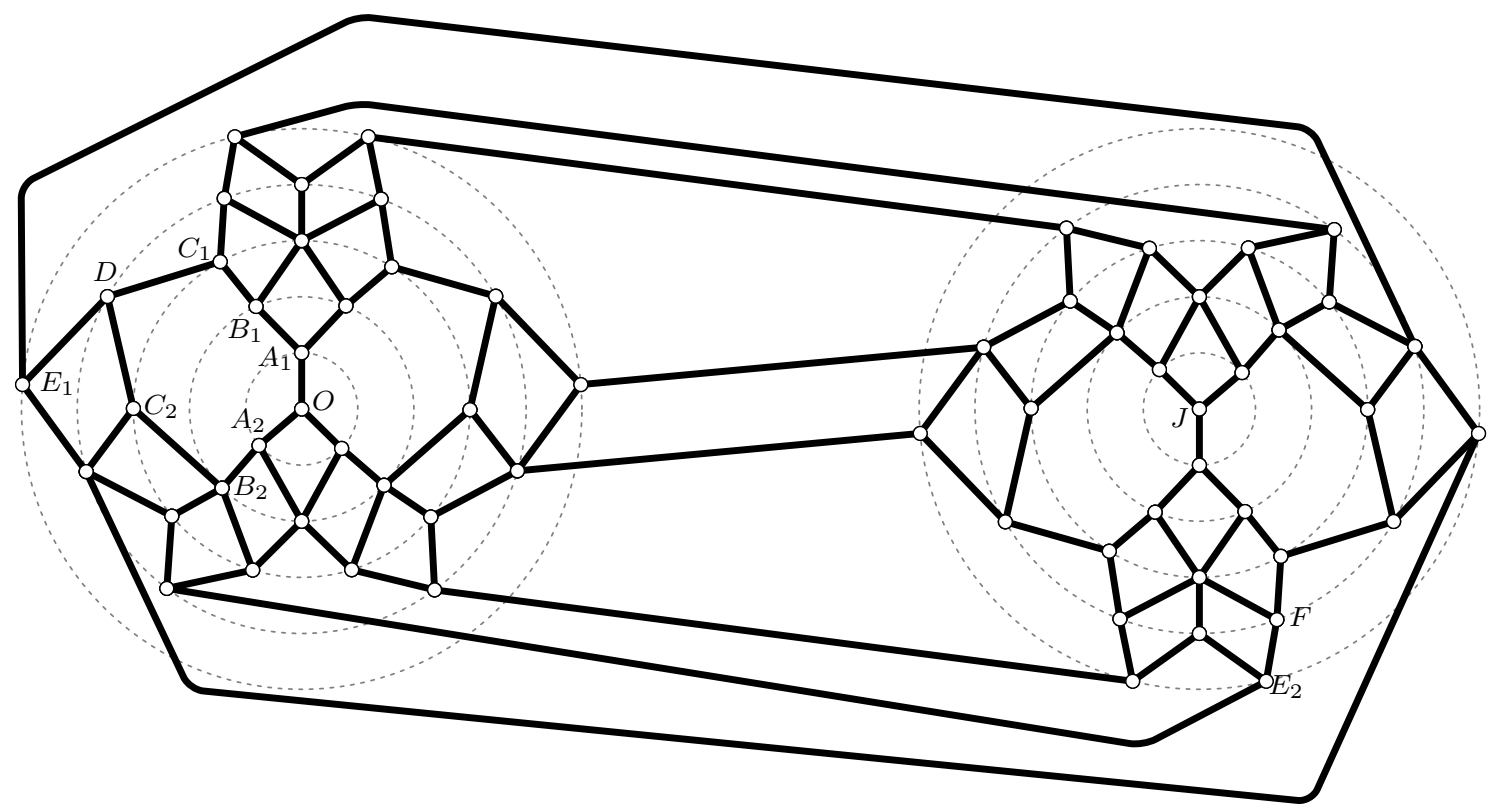

Figure 3: The graph $\widehat{G}\left(\mathrm{w}_{\mathbf{0}}^{4}\right)$.

each of the vertices $A, B, C$ is connected to all the vertices $D, E, F$ (see Figure 4). In particular, the class [132134321354321] of $E$ is connected by a long braid relation to both classes $A$ and $B$, and the class [123214323543212] of $E$ is connected to the class $D$. The class [123214321543214] of $F$ is connected to class $B$, and the class [132132432543212] of $F$ is connected to both classes $A$ and $C$.

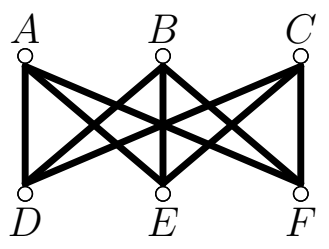

Figure 4: A $K_{3,3}$ minor of $\widehat{G}\left(\mathrm{w}_{\mathbf{0}}^{5}\right)$.

\section{Commutation Classes}

In this section, we define a family of reduced words for $w_{\mathbf{0}}$ generated by a concatenation of monotone words of lengths $n, \ldots, 2,1$, and indexed by binary vectors of length $n-1$. This family contains, as a particular case, the reduced word $w_{0}=1(21)(321) \cdots(n \cdots 21)$. 
To simplify notation, whenever $1 \leqslant a<b \leqslant n$ define the words

$$
\begin{aligned}
t_{a, b}^{+} & :=a(a+1) \cdots(b-1) b, \quad \text { and } \\
t_{a, b}^{-} & :=b(b-1) \cdots(a+1) a .
\end{aligned}
$$

The words $t_{a, b}^{+}$and $t_{a, b}^{-}$are reduced because they have no repeated letters.

Definition 17. Given a binary vector $\mathfrak{b}=\left(\mathfrak{b}_{1}, \mathfrak{b}_{2}, \ldots, \mathfrak{b}_{n-1}\right)$, with $\mathfrak{b}_{i} \in\{+,-\}$ for all $i=1,2, \ldots, n-1$, we construct the word

$$
w^{\mathfrak{b}}=w_{n}^{\mathfrak{b}_{1}} w_{n-1}^{\mathfrak{b}_{2}} \cdots w_{2}^{\mathfrak{b}_{n-1}} w_{1}
$$

where each $w_{n-\ell+1}^{\mathfrak{b}_{\ell}}$ is a monotone subword of length $n-\ell+1$ defined recursively as follows (we set $w_{1}=w_{1}^{\mathfrak{b}_{n}}$, with $\mathfrak{b}_{n}=+$ ):

- $w_{n}^{\mathfrak{b}_{1}}=\left\{\begin{array}{ll}t_{1, n}^{+}=12 \cdots n, & \text { if } \mathfrak{b}_{1}=+ \\ t_{1, n}^{-}=n \cdots 21, & \text { if } \mathfrak{b}_{1}=-\end{array}\right.$.

- For $\ell=1, \ldots, n-1$,

- if $\mathfrak{b}_{\ell}=+$ and $w_{n-\ell+1}^{\mathfrak{b}_{\ell}}=i(i+1) \cdots(j-1) j$ with $i<j$, then set

$$
w_{n-\ell}^{\mathfrak{b}_{\ell+1}}=\left\{\begin{array}{ll}
t_{i, j-1}^{+}=i(i+1) \cdots(j-1), & \text { if } \mathfrak{b}_{\ell+1}=+ \\
t_{i, j-1}^{-}=(j-1) \cdots(i+1) i, & \text { if } \mathfrak{b}_{\ell+1}=-
\end{array},\right.
$$

- if $\mathfrak{b}_{\ell}=-$ and $w_{n-\ell+1}^{\mathfrak{b}_{\ell}}=j(j-1) \cdots(i+1) i$ with $i<j$, then set

$$
w_{n-\ell}^{\mathfrak{b}_{\ell+1}}=\left\{\begin{array}{ll}
t_{i+1, j}^{+}=(i+1) \cdots(j-1) j, & \text { if } \mathfrak{b}_{\ell+1}=+ \\
t_{i+1, j}^{-}=j(j-1) \cdots(i+1), & \text { if } \mathfrak{b}_{\ell+1}=-
\end{array} .\right.
$$

Note that each word $w_{n-\ell}^{\mathfrak{b}_{\ell+1}}$ is obtained by removing the rightmost letter from $w_{n-\ell-1}^{\mathfrak{b}_{\ell}}$, and sorting the remaining letters by increasing or decreasing order according to the sign $\mathfrak{b}_{\ell+1}$. Also, note that in Definition 17, the sign $\mathfrak{b}_{n}$ is irrelevant for the construction $w_{1}=w_{1}^{\mathfrak{b}_{n}}$, that is, the letter $w_{1}^{\mathfrak{b}_{n}}$ is completely determined by the previous subword $w_{2}^{\mathfrak{b}_{n-1}}$.

Example 18. Consider the binary vector $\mathfrak{b}=(+,+,-,+,-)$ of length 5 . We then construct the subwords

$$
\begin{aligned}
& w_{6}^{\mathfrak{b}_{1}}=w_{n}^{+}=123456, \\
& w_{5}^{\mathfrak{b}_{2}}=w_{5}^{+}=12345, \\
& w_{4}^{\mathfrak{b}_{3}}=w_{4}^{-}=4321, \\
& w_{3}^{\mathfrak{b}_{4}}=w_{3}^{+}=234, \\
& w_{2}^{\mathfrak{b}_{5}}=w_{2}^{-}=32, \\
& w_{1}=3 .
\end{aligned}
$$

Thus, the word indexed by $\mathfrak{b}$ is $w^{\mathfrak{b}}=123456 \cdot 12345 \cdot 4321 \cdot 234 \cdot 32 \cdot 3$. 
Let $\mathcal{O}(n)=\left\{w^{\mathfrak{b}}: \mathfrak{b} \in\{+,-\}^{n-1}\right\}$ denote the set of all words indexed by binary vectors of length $n-1$, constructed in Definition 17. The words of this set will be designated ordered words, and are formed by subwords $w_{n-\ell}^{\mathfrak{b}_{\ell+1}}$ in decreasing order of their lengths. They will serve as a basis for the construction of a larger class of commutation classes in $\widehat{G}\left(\mathrm{w}_{0}\right)$.

Proposition 19. Any ordered word $w \in \mathcal{O}(n)$ is a reduced word for $\mathbf{w}_{\mathbf{0}}$.

Proof. Let $w^{\mathfrak{b}} \in \mathcal{O}(n)$, with $\mathfrak{b}$ a binary vector of length $n-1$. Then, the length of $w^{\mathfrak{b}}$ is the sum of the lengths of the monotone subwords $w_{n-\ell+1}^{\mathfrak{b}_{\ell}}$, for $\ell=1,2 \ldots, n$. That is

$$
\ell\left(w^{\mathfrak{b}}\right)=\sum_{\ell=1}^{n}(n-\ell+1)=\frac{(n+1) n}{2}=\ell\left(\mathbf{w}_{\mathbf{0}}\right) .
$$

Let us now prove that the permutation associated with $w^{\mathfrak{b}}$ is the longest permutation $\mathbf{w}_{\mathbf{0}} \in \mathfrak{S}_{n+1}$. Assume that $\mathfrak{b}_{1}=-$. The permutation associated with $w_{n}^{-}=n \cdots 21$ has one-line notation $23 \cdots(n+1) 1$. On the other hand, the permutation associated with $w_{n-1}^{\mathfrak{b}_{2}} \cdots w_{2}^{\mathfrak{b}_{n-1}} w_{1}$ leaves the letter 1 fixed, and only acts on the set $\{2, \ldots, n+1\}$. By induction, the permutation associated with $w_{n-1}^{\mathfrak{b}_{2}} \cdots w_{2}^{\mathfrak{b}_{n-1}} w_{1}^{+}$is the longest permutation on the set $\{2, \ldots, n+1\}$, and it follows that the permutation associated with $w^{\mathfrak{b}}$ is the longest permutation of $\mathfrak{S}_{n+1}$.

The proof is similar if $\mathfrak{b}_{1}=+$. Therefore $w^{\mathfrak{b}}$ is a reduced word for $\mathbf{w}_{\mathbf{0}}$.

Proposition 20. The set $\mathcal{O}(n)$ contains $2^{n-1}$ distinct words, each belonging to a different commutation class.

Proof. By construction, there are a total of $2^{n-1}$ words $w^{\mathfrak{b}}$, with $\mathfrak{b}$ a binary vector of length $n-1$, and they are all distinct. We prove by induction on $n \geqslant 2$ that any two of these words are in distinct commutation classes.

When $n=2$ there are only two words $w^{(+)}=12 \cdot 1$ and $w^{(-)}=21 \cdot 2$, which clearly are not in the same commutation class. Suppose now $n>2$, and consider words $w^{\mathfrak{b}}$ and $w^{\mathfrak{b}^{\prime}}$ with $\mathfrak{b}_{1}=+$ and $\mathfrak{b}_{1}^{\prime}=-$. Then, $T\left(w^{\mathfrak{b}}, x y z\right)=1$ for any integers $y<z$ with $x=1$, while $T\left(w^{\mathfrak{b}^{\prime}}, x y z\right)=-1$ for any integers $x<y$ and $z=n+1$. Thus, $T\left(w^{\mathfrak{b}}, 1 y(n+1)\right) \neq T\left(w^{\mathfrak{b}^{\prime}}, 1 y(n+1)\right)$, for any $2 \leqslant y \leqslant n$, showing, by Lemma 10 , that the commutation classes of $w^{\mathfrak{b}}$ and $w^{\mathfrak{b}^{\prime}}$ are distinct. Consider next the case $\mathfrak{b}_{1}=\mathfrak{b}_{1}^{\prime}$. Then, we can write $w^{\mathfrak{b}}=w_{n}^{\mathfrak{b}_{1}} \cdot u^{\mathfrak{d}}$ and $w^{\mathfrak{b}^{\prime}}=w_{n}^{\mathfrak{b}_{1}} \cdot u^{\mathfrak{d}^{\prime}}$, where $\mathfrak{d}=\left(\mathfrak{b}_{2}, \ldots, \mathfrak{b}_{n-1}\right)$ and $\mathfrak{d}^{\prime}=\left(\mathfrak{b}_{2}^{\prime}, \ldots, \mathfrak{b}_{n-1}^{\prime}\right)$. By the inductive step, we find that the words $u^{\mathfrak{d}}$ and $u^{\mathfrak{d}^{\prime}}$ are in distinct commutation classes, showing that $w^{\mathfrak{b}}$ and $w^{\mathfrak{b}^{\prime}}$ are in distinct commutation classes.

We can use short and long braid relations in an ordered word $w^{\mathfrak{b}}$ to "move" a factor $w_{n-\ell+1}^{\mathfrak{b}_{\ell}}$ within the other factors of $w^{\mathfrak{b}}$, according to the rules of the following lemma. These rules will be used to construct two classes of words: atoms, that is reduced words with no short braid relations, and alternating words, which can be interpreted as fillings of certain moon-polyomino shapes. 
Lemma 21. Given positive integers $a<k \leqslant b$, consider the increasing word $w^{+}=$ $a(a+1) \cdots(b-1) b$ and the decreasing word $w^{-}=b(b-1) \cdots(a+1) a$. Then,

1. $\left[k \cdot w^{+}\right] \underset{\mathrm{L}}{\sim}\left[w^{+} \cdot(k-1)\right]$.

2. $\left[(k-1) \cdot w^{-}\right] \sim\left[w^{-} \cdot k\right]$.

For instance, the following list of reduced words for $w_{\mathbf{0}}$ shows how we can use short and long braid relations to "move" the factor $w_{3}^{+}=234$ of $w^{\mathfrak{b}}$ in Example 18, to the leftmost position:

$$
\begin{aligned}
& 123456 \cdot 12345 \cdot 4321 \cdot \mathbf{2 3 4} \cdot 32 \cdot 3=w^{\mathfrak{b}} \\
& 123456 \cdot 12345 \cdot \mathbf{1 2 3} \cdot 4321 \cdot 32 \cdot 3 \\
& 123456 \cdot \mathbf{2 3 4} \cdot 12345 \cdot 4321 \cdot 32 \cdot 3 \\
& \mathbf{3 4 5} \cdot 123456 \cdot 12345 \cdot 4321 \cdot 32 \cdot 3
\end{aligned}
$$

Given an ordered word $w^{\mathfrak{b}}$, and a partition $I \cup J$ of $[n-1]$, we construct the two side ordered word $w_{I}^{\mathfrak{b}}$ as the concatenation

$$
\prod_{i \in I} \underline{w}_{i}^{\mathfrak{b}_{n-i+1}} \cdot w_{n}^{\mathfrak{b}_{1}} \cdot \prod_{j \in J} w_{j}^{\mathfrak{b}_{n-j+1}}
$$

where each letter of $\underline{w}_{i}^{\mathfrak{b}_{n-i+1}}$ is obtained from the corresponding letter of $w_{i}^{\mathfrak{b}_{n-i+1}}$ by adding the value $\sum_{k=i+1}^{n} \mathfrak{b}_{k}$, considering the signals + and - as 1 and -1 , respectively. The factor $\underline{w}_{i}^{\mathfrak{b}_{n-i+1}}$ can also be seen as obtained from the factor $w_{i}^{\mathfrak{b}_{n-i+1}}$ of $w^{\mathfrak{b}}$ by the application of short and long braid relations, according to the rules of Lemma 21. Moreover, in $\prod_{i \in I} \underline{w}_{i}^{\mathfrak{b}_{n-i+1}}$ the factors are written from left to right in increasing order of their lengths, and in $\prod_{j \in J} w_{j}^{\mathfrak{b}_{n-j+1}}$ the factors are written from left to right in decreasing order of their lengths.

For example, the word (4) can be written as $w_{I}^{\mathfrak{b}}$, where $b=(+,+,-,+,-)$ and $I=\{3\}$, where $\underline{w}_{3}^{+}=345$.

Definition 22. Let $\mathcal{T} \mathcal{S O}(n)=\left\{w_{I}^{\mathfrak{b}}: w^{\mathfrak{b}} \in \mathcal{O}(n)\right.$ and $\left.I \subseteq[n-1]\right\}$ denote the set of all two side ordered words.

Note that each word in $\mathcal{T S O}(n)$ is a reduced word for $w_{\mathbf{0}}$, since it is obtained from some ordered word in $\mathcal{O}(n)$, which by Proposition 19 is reduced, by applying short and long braid relations.

Lemma 23. The set $\mathcal{T S O}(n)$ contains $3 \times 4^{n-2}$ distinct words.

Proof. To construct a two side ordered word, there are two possibilities for the sign $\mathfrak{b}_{1}$ of $w_{n}^{\mathfrak{b}_{1}}$, and two possibilities for whether $w_{1}$ belongs to the set $I$ or not. For all other factors there are four possible choices, two for the signal and two for whether it belongs to $I$ or not. This amounts to $4^{n-1}$ possibilities. Note however, that if $\mathfrak{b}_{1}$ and $\mathfrak{b}_{2}$ have distinct signs, then $w_{n}^{\mathfrak{b}_{1}} w_{n-1}^{\mathfrak{b}_{2}}=\underline{w}_{n-1}^{\mathfrak{b}_{1}} w_{n}^{\mathfrak{b}_{2}}$. Thus, there are a total of $4^{n-1}-4^{n-2}=3 \times 4^{n-2}$ distinct two side ordered words in $\mathcal{T S O}(n)$. 


\subsection{Atoms}

Definition 24. A reduced word $w \in R\left(\mathbf{w}_{\mathbf{0}}\right)$ whose commutation class contains only itself is called an atom of $\widehat{G}\left(\mathrm{w}_{\mathbf{0}}\right)$.

Clearly, a reduced word $w \in R\left(\mathrm{w}_{\mathbf{0}}\right)$ is an atom if and only if each factor $i j$ of length 2 of $w$ is formed by consecutive letters, i.e. $|i-j|=1$. We will show that there are exactly four atoms in $\widehat{G}\left(\mathbf{w}_{\mathbf{0}}\right)$, for $n \geqslant 3$, namely the words $w^{\mathfrak{b}}, w^{-\mathfrak{b}},\left(w^{\mathfrak{b}}\right)^{\mathrm{R}}$ and $\left(w^{-\mathfrak{b}}\right)^{\mathrm{R}}$, with $\mathfrak{b}=(+,-,+,-, \ldots)$.

Lemma 25. Let $a, b$ be integers such that $a<b-1$. Then, the words

1. $(a+1) \cdot t_{a, b}^{+} \cdot t_{a, b-1}^{-} \cdot(a+1), \quad$ and

2. $t_{a, b}^{+} \cdot t_{a, b-1}^{-} \cdot t_{a+1, b}^{+}$.

are not reduced.

Proof. The word $(a+1) \cdot t_{a, b}^{+} \cdot t_{a, b-1}^{-} \cdot(a+1)$ has $2(b-a)+3$ letters, and the corresponding permutation

$$
\mathbf{w}=12 \cdots(a-1) \cdot(b+1)(a+1)(a+2) \cdots(b-1) a \cdot(b+2)(b+3) \cdots n(n+1),
$$

has, by $(3)$, length $\ell(\mathrm{w})=2(b-a)+1$. It follows that $(a+1) \cdot t_{a, b}^{+} \cdot t_{a, b-1}^{-} \cdot(a+1)$ is not reduced. Similarly, the word $t_{a, b}^{+} \cdot t_{a, b-1}^{-} \cdot t_{a+1, b}^{+}$has $3(b-a)+1$ letters, and the corresponding permutation

$$
\mathbf{v}=12 \cdots(a-1) \cdot b(b+1)(a+1)(a+2) \cdots(b-1) a \cdot(b+2)(b+3) \cdots n(n+1),
$$

has length $\ell(\mathrm{v})=3(b-a)-1$. Thus, $t_{a, b}^{+} \cdot t_{a, b-1}^{-} \cdot t_{a+1, b}^{+}$is not reduced.

Lemma 26. The increasing word $12 \cdots n$ is the only reduced word amongst the set of all words of length $\geqslant n$ over the alphabet $[n]$, having leftmost letter 1 and rightmost letter $n$, and where each factor of length 2 is formed by consecutive letters.

Proof. Let $k$ be the length of a word $u$ in the conditions of the lemma. We start by noticing that if $k=n$, then the increasing word $u=12 \cdots n$ is reduced, since by (3) the corresponding permutation $(n+1) 12 \cdots n$ has length $n$. Assuming now that $k>n$, the word $u$ is a concatenation of increasing factors with decreasing factors. Since $u_{1}=1$ and $u_{k}=n, u$ must have a factor of the form

$$
t_{a, b}^{+} \cdot t_{a, b-1}^{-} \cdot t_{a+1, b}^{+},
$$

for some integers $a<b$, which by Lemma 25 is not reduced. It follows that $u$ is not reduced.

Theorem 27. For $n \geqslant 3$, there are exactly 4 atoms in the graph $\widehat{G}\left(\mathbf{w}_{\mathbf{0}}\right)$. 
Proof. We start by exhibiting the four atoms of $\widehat{G}\left(\mathrm{w}_{\mathbf{0}}\right)$. Let $\mathfrak{b}=(+,-,+,-, \ldots)$ and $-\mathfrak{b}=(-,+,-,+, \ldots)$ be the binary vectors of length $n-1$ having alternating signs, and consider the corresponding ordered words $w^{\mathfrak{b}}$ and $w^{-\mathfrak{b}}$ in $\mathcal{O}(n)$ :

$$
w^{\mathfrak{b}}=w_{n}^{+} w_{n-1}^{-} w_{n-2}^{+} \cdots w_{1} \text { and } w^{-\mathfrak{b}}=w_{n}^{-} w_{n-1}^{+} w_{n-2}^{-} \cdots w_{1}=\left(w^{\mathfrak{b}}\right)^{\bullet} .
$$

By Proposition 19, $w^{\mathfrak{b}}$ and $w^{-\mathfrak{b}}$ are reduced words for $\mathbf{w}_{\mathbf{0}}$, and each factor of length 2 is formed by consecutive letters. It follows that $w^{\mathfrak{b}}$ and $w^{-\mathfrak{b}}$ are atoms of $\widehat{G}\left(\mathbf{w}_{\mathbf{0}}\right)$. Similarly, each factor of length 2 of the reduced words $\left(w^{\mathfrak{b}}\right)^{\mathrm{R}}$ and $\left(w^{-\mathfrak{b}}\right)^{\mathrm{R}}$, is formed by consecutive letters, showing that they are also atoms of $\widehat{G}\left(\mathrm{w}_{\mathbf{0}}\right)$. For $n \geqslant 3$, these four atoms are all distinct.

We will prove next that these four words are the only atoms in $\widehat{G}\left(\mathrm{w}_{\mathbf{0}}\right)$. Let $w \in R\left(\mathrm{w}_{\mathbf{0}}\right)$ be an atom. Since the sets of letters appearing in any two reduced words for $w \in \mathfrak{S}_{n+1}$ are the same (see [4]), a reduced word for $\mathbf{w}_{\mathbf{0}}$ must have the letters 1 and $n$. By Lemma 26, $w$ must have as a factor $t_{1, n}^{+}=12 \cdots n$ or $t_{1, n}^{-}=n \cdots 21$, since any factor of length 2 of $w$ consists of consecutive letters. Suppose the first case happens (the other is analogous).

If $w$ has two 1 s and two $n$ s, then one $n$ must be on the left side of the factor $t_{1, n}^{+}$, since otherwise we would have a factor of length greater than $n$ starting with 1 and ending with $n$, which by Lemma 26 is not reduced. Again by Lemma 26, this means that $w$ must have the factor $t_{2, n}^{-} \cdot t_{1, n}^{+}$. But then, if 1 is either on the left side or on the right side of $t_{2, n}^{-} \cdot t_{1, n}^{+}$, then $w$ has a factor of length greater than $n$ starting with 1 and ending with $n$, or vice-versa, which by Lemma 26 is not reduced. Thus, $w$ has at least two 1 s and one $n$, or one 1 and at least two $n$ s. Notice that one of these cases must occur, since otherwise we would have

$$
w=w^{\prime} \cdot(12 \cdots n) \cdot w^{\prime \prime},
$$

with $w^{\prime}$ and $w^{\prime \prime}$ words over the alphabet $\{2, \ldots, n-1\}$. In this case, the permutation associated with $w^{\prime}$ fixes 1 and $n+1$, and then the permutation associated with $w^{\prime} \cdot(12 \cdots n)$ sends 1 to $n+1$ and $n+1$ to $n$. Therefore, $w$ cannot be a word for $\mathbf{w}_{\mathbf{0}}$ since it does not send $n+1$ to 1 , contradicting the definition of $w$. Then, $w$ must have a factor

$$
t_{1, n}^{+} \cdot t_{1, n-1}^{-} \cdot t_{2, k}^{+} \quad \text { or } \quad t_{1, k}^{-} \cdot t_{2, n}^{+} \cdot t_{1, n-1}^{-},
$$

for some $k<n$. Assume the former case (the other is analogous). Then, this must be the leftmost factor of $w$, since otherwise $w$ would have the factor $2 \cdot t_{1, n}^{+} \cdot t_{1, n-1}^{-} \cdot 2$, which by Lemma 25 is not reduced.

Since $k<n$, the next factor in $w$ must be a decreasing sequence $t_{i, k-1}^{-}$for some integer $i$. For the same reason as before $i$ is must be bigger than 1, otherwise $w$ would have the factor $t_{1, k}^{-} \cdot t_{2, k}^{+} \cdot t_{1, k-1}^{-}$, which is not reduced. Consequently, the new factor should be shorter (in length) than the previous one. Repeating the same reasoning, $w$ is formed by a sequence of factors in decreasing order of lengths, alternating between increasing and decreasing factors. So it has, at most, $n$ factors and, to be reduced, these lengths add up to $\frac{(n+1) n}{2}$. Therefore, these lengths must be, respectively, $n,(n-1), \ldots, 1$.

Therefore, $w$ is the atom $w^{\mathfrak{b}}$ that we have constructed above. The different choices we can made in the proof gives the other three atoms. 
Using Lemma 21, it is easy to see that $\left(w^{\mathfrak{b}}\right)^{\mathrm{R}}=w_{[n-1]}^{-\mathfrak{b}}$ and $\left(w^{-\mathfrak{b}}\right)^{\mathrm{R}}=w_{[n-1]}^{\mathfrak{b}}$, showing that the four atoms $w^{\mathfrak{b}}, w^{-\mathfrak{b}},\left(w^{\mathfrak{b}}\right)^{\mathrm{R}}$ and $\left(w^{-\mathfrak{b}}\right)^{\mathrm{R}}$ are words in $\mathcal{T} \mathcal{S O}(n)$.

\subsection{Alternating classes}

Definition 28. A word $w$ over the alphabet $\{a, b\}$ is alternating if it is of the form $(a b)^{k}$ or $(a b)^{k} a$ for some integer $k \geqslant 0$. A word $w$ over the alphabet $[n]$ is alternating if each subword $w_{\mid\{i, i+1\}}$ of $w$, formed only by the letters $i$ and $i+1$, is alternating, for $i=1,2, \ldots, n-1$.

It follows that the difference between the number of letters $i$ and $i+1 \mathrm{in}$ an alternating word $w$ is at most one. Moreover, between two consecutive letters $i$ in $w$ there is exactly one letter $i-1$ and exactly one letter $i+1$. Denote by $A R\left(\mathrm{w}_{\mathbf{0}}\right)$ the set of all alternating reduced words in $R\left(\mathrm{w}_{\mathbf{0}}\right)$. We will characterize all commutation classes in $A R\left(\mathrm{w}_{\mathbf{0}}\right)$.

Proposition 29. Let $w \in A R\left(w_{\mathbf{0}}\right)$ be an alternating word, and $i \in\{2, \ldots, n\}$. Then, $T(w, x i y)$ is constant for all triples $x<i<y$.

Proof. Suppose the letter $i$ occurs in $w$ to the left of the first letter $i-1$ (the other case is analogous), that is $w_{\mid\{i-1, i\}}=(i(i-1))^{k}$ or $w_{\mid\{i-1, i\}}=(i(i-1))^{k} i$, for some integer $k$. Then, by the successive action of the generators of $w$ on the identity permutation, the first inversion $(a, b)$ with $a<b$ and $i \in\{a, b\}$, on the succession of permutations from the identity to $\mathbf{w}_{\mathbf{0}}$, occurs with a pair $(i, y)$ with $i<y$. Thus, the integer $i$, after this inversion, is in position $i+1$, and only the generators $i$ and $i+1$ can affect it. Since $w$ is an alternating word, there is a letter $i+1$ before the next letter $i$ in $w$, which means that the next inversion containing the integer $i$ is again with an integer $y^{\prime}$ such that $i<y^{\prime}$. This process is repeated until each pair of integers $(i, t)$, with $i<t$, is an inversion. It follows that $T(w, x i y)=-1$.

We now define a subset $V$ of $\mathcal{T S O}(n)$, whose elements are alternating words. Moreover, we will prove that the minimum element, in lexicographic order, of any alternating class is an element of the set $V$.

Definition 30. Let $V$ be the set of words

$$
w_{I}^{-}=\prod_{i \in I} \underline{w}_{i}^{-} \cdot w_{n}^{-} \cdot \prod_{j \in J} w_{j}^{-} \in \mathcal{T} \mathcal{S O}(n),
$$

where - in $w_{I}^{-}$stands for the binary vector $(-)^{n-1}$ of length $n-1$, and $I \cup J$ is any partition of $[n-1]$.

According to Definition 17, we have

$$
\underline{w}_{i}^{-}=i(i-1) \cdots 21 \quad \text { and } \quad w_{j}^{-}=n(n-1) \cdots(n-j+2)(n-j+1),
$$

for $i \in I$ and $j \in J$, and thus $w_{I}^{-} \in A R\left(\mathrm{w}_{\mathbf{0}}\right)$. 
Lemma 31. The set $V$ is a subset of $A R\left(w_{\mathbf{0}}\right)$ with cardinality $2^{n-1}$. Moreover, the commutation classes of any two distinct words of $V$ are distinct.

Proof. By construction, each word $w_{I}^{-}$, with $I \subseteq[n-1]$, is the minimum element, in lexicographic order, of its class, since they are formed by the concatenation of strictly decreasing subwords, with the rightmost letter of a subword strictly smaller than the leftmost letter of the subword sitting on its right. Therefore, each word in $V$ is in a different commutation class, and the number of words in $V$ is the number of subsets of $[n-1]$.

Example 32. For $n+1=6$, the set $V$ is formed by the $2^{4}$ words below:

$$
\begin{array}{cc}
w_{\emptyset}^{-}=54321 \cdot 5432 \cdot 543 \cdot 54 \cdot 5, & w_{\{1\}}^{-}=1 \cdot 54321 \cdot 5432 \cdot 543 \cdot 54, \\
w_{\{1,2\}}^{-}=1 \cdot 21 \cdot 54321 \cdot 5432 \cdot 543, & w_{\{1,3\}}^{-}=1 \cdot 321 \cdot 54321 \cdot 5432 \cdot 54, \\
w_{\{1,4\}}^{-}=1 \cdot 4321 \cdot 54321 \cdot 543 \cdot 54, & w_{\{2\}}^{-}=21 \cdot 54321 \cdot 5432 \cdot 543 \cdot 5, \\
w_{\{2,3\}}^{-}=21 \cdot 321 \cdot 54321 \cdot 5432 \cdot 5, & w_{\{2,4\}}^{-}=21 \cdot 4321 \cdot 54321 \cdot 543 \cdot 5, \\
w_{\{3\}}^{-}=321 \cdot 54321 \cdot 5432 \cdot 54 \cdot 5, & w_{\{3,4\}}^{-}=321 \cdot 4321 \cdot 54321 \cdot 54 \cdot 5, \\
w_{\{4\}}^{-}=4321 \cdot 54321 \cdot 543 \cdot 54 \cdot 5, & w_{\{1,2,3\}}^{-}=1 \cdot 21 \cdot 321 \cdot 54321 \cdot 5432, \\
w_{\{1,2,4\}}^{-}=1 \cdot 21 \cdot 4321 \cdot 54321 \cdot 543, & w_{\{1,3,4\}}^{-}=1 \cdot 321 \cdot 4321 \cdot 54321 \cdot 54, \\
w_{\{2,3,4\}}^{-}=21 \cdot 321 \cdot 4321 \cdot 54321 \cdot 5, & w_{\{1,2,3,4\}}^{-}=1 \cdot 21 \cdot 321 \cdot 4321 \cdot 54321 .
\end{array}
$$

Notice that the word $w_{0}=w_{[n-1]}^{-} \in V$. As a particular case of Proposition 29, we get the following result.

Corollary 33. Let $w_{I}^{-} \in V$. Then, the value of $T\left(w_{I}^{-}\right.$, xiy) is 1 (respectively -1) if $i-1 \in I$ (respectively $i-1 \notin I$ ), for all integer $x<i<y$.

We will show next that each alternating reduced word in $A R\left(\mathrm{w}_{\mathbf{0}}\right)$ is in a class of an element of $V$.

Theorem 34. There are exactly $2^{n-1}$ commutation classes in $A R\left(w_{\mathbf{0}}\right)$.

Proof. By Lemma 10, the commutation class of $w \in R\left(\mathrm{w}_{\mathbf{0}}\right)$ is characterized by the values of $T(w, a b c)$, for all triples $a<b<c$. Proposition 29 shows that it is enough to know the values of $T(w, k(k+1)(k+2))$, for $k=1, \ldots, n-1$. Thus, there are at most $2^{n-1}$ commutation classes in $A R\left(\mathrm{w}_{\mathbf{0}}\right)$. Lemma 31 shows that there are exactly $2^{n-1}$ such classes.

\subsection{Complete moon-polyominoes}

Next we give an interpretation of alternating words as standard fillings of certain moonpolyomino Young tableaux.

A diagram $\delta$ is a finite subset of the two-dimensional integer lattice $\mathbb{Z}^{2}$, which we identify with a set of cells in the plane, using the English convention for the coordinates of each cell, i.e. matrix-like coordinates. The number $|\delta|$ of cells in the diagram is the 
size of $\delta$. A column of $\delta$ is the set of cells along a vertical line, and a row is the set of cells along a horizontal line. A diagonal $D_{k}$ of a diagram $\delta$, with $k \in \mathbb{Z}$, is the set $D_{k}=\{(i, j) \in \delta: i-j=k\}$.

A diagram $\delta$ is convex if for any two cells in a either column or row, the elements of $\mathbb{Z}^{2}$ in between are also cells of the diagram. It is intersection-free if any two columns are comparable, i.e. the set of row coordinates of cells in one column is contained in the set of row coordinates of cells in the other. For example, the first diagram in Figure 5 is convex but not intersection-free, the second is neither convex nor intersection-free, while the third is a convex intersection-free diagram.
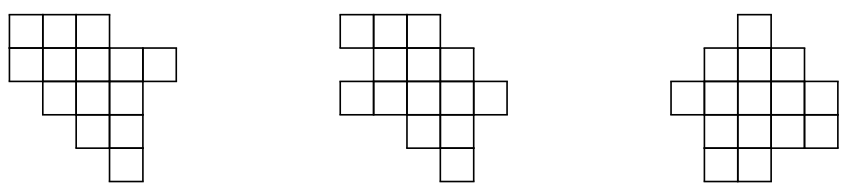

Figure 5: Example of diagrams.

Definition 35. A moon-polyomino is a convex intersection-free diagram. A moonpolyomino with exactly $n$ columns is said to be an $n$-diagonal moon-polyomino if it has a column of length $i$, for all $i \in[n]$, and exactly $n$ diagonals.

For instance, the third diagram in Figure 5 is a moon-polyomino but not a 5 -diagonal moon-polyomino. Note that since an $n$-diagonal moon-polyomino is convex and intersection-free, all columns on the right side of the column of length $n$ are arranged in decreasing order of their lengths, from left to right. Moreover, the top box of each one of these columns are in the same diagonal. We call this set of columns, including the column of length $n$, the right side of the moon-polyomino. Similarly, all columns on the left side of the column of length $n$ are arranged in increasing order of their lengths, from left to right, and the bottom box of each one of these columns are in the same diagonal. We call these set of columns, excluding the column of length $n$, the left side of the moon-polyomino. Analogously we define the up side and down side of a moon-polyomino as the subdiagram formed by all rows including and below the row of length $n$, and as the diagram formed by all rows above the row of length $n$, respectively.

The shape of an $n$-diagonal moon-polyomino is completely determined by the sequence of its column lengths, and thus it is identified by that sequence. For example, the shape of the last 5-diagonal moon-polyomino in Figure 6 is $(1,5,4,3,2)$.

Proposition 36. There are exactly $2^{n-1} n$-diagonal moon-polyominoes.

Proof. An $n$-diagonal moon-polyomino is completely characterized by choosing on which side of the moon-polyomino the column of length $i$ will be, for each $i \in[n-1]$.

Figure 6 shows the $2^{4}$ 5-diagonal moon-polyominoes.

A tableau $P$ of shape $\delta$ is an assignment of integers to the cells of $\delta$. If the entries of the cells of $P$ are the integers in $[|\delta|]=\{1,2, \ldots,|\delta|\}$, used exactly once, the tableau is called 

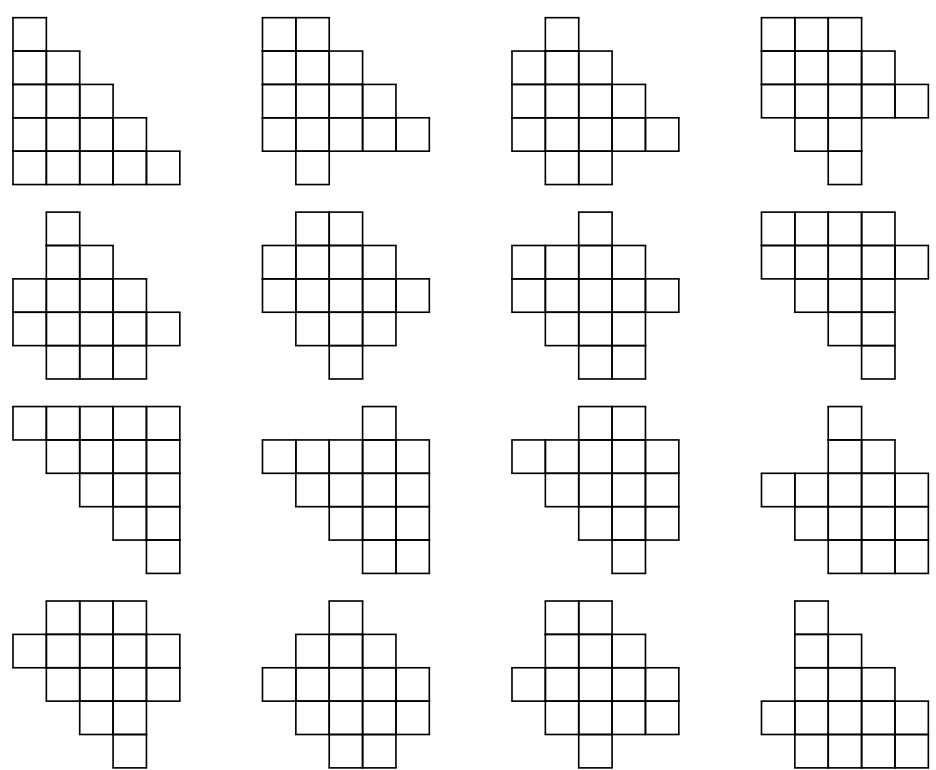

Figure 6: The 5-diagonal moon-polyominoes.

standard. A Young tableau is a tableau in which the entries are increasing down columns, and across rows, from left to right. A standard Young tableau (SYT) is a Young tableau which is also a standard tableau. Figure 7 shows a SYT of the 5-diagonal moon-polyomino of shape $(1,3,5,4,2)$. Stanley [12] proved that the cardinality of $R\left(\mathrm{w}_{\mathbf{0}}\right)$ is given by the number of all SYT with partition shape $(n, n-1, \ldots, 1)$.

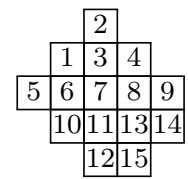

Figure 7: SYT of 5-diagonal moon-polyomino shape

The number of SYT of shape $\delta$ is invariant under reflection in a diagonal line $(i, j) \mapsto$ $(j, i)$ or $(i, j) \mapsto(-j,-i)$, and reflection in the origin $(i, j) \mapsto(-i,-j)$, that is rotation by $180^{\circ}$ [1], which is the composition of the other two reflections.

Let $w=w_{1} \cdots w_{\ell} \in A R\left(\mathbf{w}_{\mathbf{0}}\right)$ be an alternating word with content $c(w)=\left(c_{1}, \ldots, c_{n}\right)$. We assign to $w$ a tableau $P(w)$ that we will show is a SYT of $n$-diagonal moon-polyomino shape. This tableau is constructed by the overlapping of $n$ diagonals $D_{i-1}$, where each diagonal $D_{i-1}$ contains the positions of the letters $i$ in $w$. The first box of $D_{i}$ is placed over or in front of the first box of $D_{i-1}$, according to whether the first occurrence of the letter $i+1$ in $w$ is before or after the first occurrence of the letter $i$.

The following algorithm encodes this procedure, by constructing a sequence of tableaux $\emptyset=P_{0}(w), P_{1}(w), \ldots, P_{n}(w)=P(w)$, where $P_{i}(w)$ is obtained from $P_{i-1}(w)$ by overlapping it with the diagonal $D_{i}$. 


\section{Algorithm 1.}

1. Start with the empty subset $P_{0}(w)$ of $\mathbb{Z} \times \mathbb{Z}$.

2. Add $c_{1}$ cells at positions $(j, j), j=1, \ldots, c_{1}$.

3. Fill these $c_{1}$ cells with the positions of the letters 1 , from left to right, in $w$. Let $P_{1}(w)$ be the resulting tableau.

4. For $i=2, \ldots, n$,

a. Let $(a, b)$ be the coordinates of the cell in $P_{i-1}(w)$ corresponding to the leftmost letter $i-1$ of $w$, and let $k$ be its label.

b. If the first occurrence of the letter $i$ in $w$ is before the $k$ th place, then

i. Add $c_{i}$ cells at positions $(a-1+j, b+j), j=0, \ldots, c_{i}-1$.

ii. Fill these $c_{i}$ cells with the positions of the letters $i$, from left to right, in $w$. Let $P_{i}(w)$ be the resulting tableau.

Else, do

i. Add $c_{i}$ cells at positions $(a+j, b+1+j), j=0, \ldots, c_{i}-1$.

ii. Fill these $c_{i}$ cells with the positions of the letters $i$, from left to right, in $w$. Let $P_{i}(w)$ be the resulting tableau.

Example 37. The successive steps in the construction of the tableau $P(w)$ corresponding to the reduced alternating word $w=253145213425312 \in A R\left(\mathrm{w}_{\mathbf{0}}\right)$ are

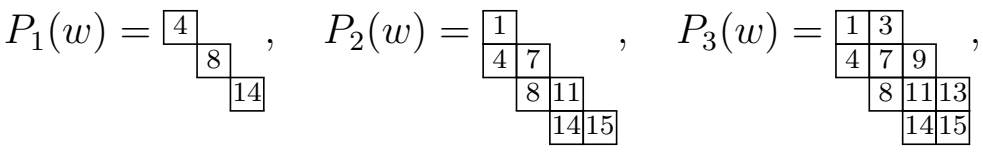

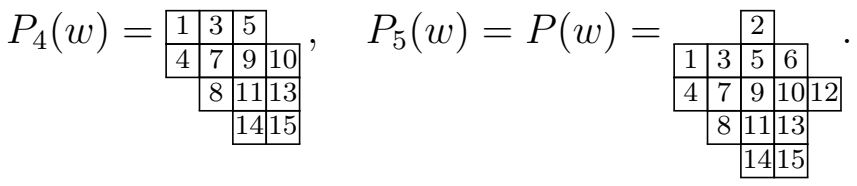

Lemma 38. If $w$ is an alternating word, then $P(w)$ is a Young tableau.

Proof. The alternating property of the subword $w_{\mid\{i, i+1\}}$, for $i \in[n-1]$, shows that any two consecutive diagonals of $P(w)$ satisfy the tableau condition, that is the entries increase along rows from left to right, and along columns, from top to bottom. It follows that $P(w)$ is a Young tableau.

Lemma 39. Let $I=\left\{i_{1}<\cdots<i_{k}\right\} \subseteq[n-1]$ and $[n] \backslash I=\left\{j_{1}<\cdots<j_{\ell}<n\right\}$. Then the shape of $P\left(w_{I}^{-}\right)$is the $n$-diagonal moon-polyomino $\left(i_{1}, \ldots, i_{k}, n, j_{\ell}, \ldots, j_{1}\right)$. 
Proof. The word $w_{I}^{-}$can be written as a product of factors $w_{I}^{-}=\underline{w}_{i_{1}}^{-} \cdots \underline{w_{i_{k}}^{-}} \cdot w_{n}^{-} \cdot w_{j_{\ell}}^{-} \cdots w_{j_{1}}^{-}$. By construction, each of these factors corresponds to a column of $P\left(w_{I}^{-}\right)$, with the number of boxes equal to the length of the corresponding factor. Since all factors $\underline{w}_{i_{q}}^{-}$end with the letter 1 , then all the columns $1,2, \cdots, k+1$ end in the diagonal $D_{0}$ associated with the letter 1 , and similarly, since all factor $w_{j_{p}}^{-}$start with the letter $n$, then the last $\ell+1$ columns start in the same diagonal $D_{n-1}$.

Since the indices in $I$ appear in $w_{I}^{-}$in increasing order, the set of row coordinates of column $q$ is contained in the set of row coordinates of column $q+1$, for $q=1, \ldots, k$, and the same happens with the last $\ell+1$ columns. This shows that the shape of $P\left(w_{I}^{-}\right)$is the $n$-diagonal moon-polyomino $\left(i_{1}, \ldots, i_{k}, n, j_{\ell}, \ldots, j_{1}\right)$.

Denote by $s(V)$ the set of shapes of the tableaux $P(v)$, with $v \in V$, and let $P V$ be the set of all Young tableaux with shapes in $s(V)$.

Theorem 40. The map $P: A R\left(w_{0}\right) \rightarrow P V$, that sends $w$ into $P(w)$, is a bijection. Moreover, $w \sim v$ if and only if $P(v)$ and $P(w)$ have the same shape.

Proof. Lemma 38 shows that $P$ is well defined. By Algorithm 1, the shape of the tableau $P(w)$, with $w \in A R\left(\mathbf{w}_{\mathbf{0}}\right)$, is completely characterized by the subwords $w_{\mid\{i, i+1\}}$ for $i \in$ $[n-1]$. It follows by Lemma 1 that given $w, v \in A R\left(\mathrm{w}_{\mathbf{0}}\right)$, we have $w \sim v$ if and only if $P(v)$ and $P(w)$ have the same shape.

Note also that the map $P$ is invertible. If $Q \in P V$, has the shape of $P\left(w_{I}^{-}\right)$, we can construct a word $w$ over the alphabet $\{1,2, \ldots, n\}$ by setting the letter $i$ in position $k$ whenever the tableau $Q$ has the integer $k$ in a box of the $D_{i-1}$ diagonal, for all $i \in[n]$. Since any two consecutive diagonals of $Q$ satisfy the Young tableau condition, it follows that each subword $w_{\mid\{i, i+1\}}$ is alternating and satisfy $w_{\mid\{i, i+1\}}=\left(w_{I}^{-}\right)_{\mid\{i, i+1\}}$ for all $i \in[n-1]$. By Lemma 1, we have $w \sim w_{I}^{-}$, and thus $w \in A R\left(\mathrm{w}_{\mathbf{0}}\right)$, with $P(w)=Q$. This proves that $P$ is a bijection and that $w \sim v$ if and only if $P(v)$ and $P(w)$ have the same shape.

Example 41. The alternating word $w=253145213425312$ in Example 37 is in the class of $w_{\{2,3\}}^{-}$since the shapes of $P\left(w_{\{2,3\}}^{-}\right)$and $P(w)$ are the same, with

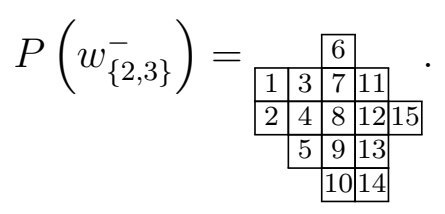

Note that the filling of $P\left(w_{\{2,3\}}^{-}\right)$is obtained by writing the integers from 1 to 15 down columns, starting from the leftmost one. The tableaux of the words in $V$ are obtained in the same manner.

Corollary 42. The map $s: V \rightarrow s(V)$ is a bijection that sends each word $v \in V$ to the shape of $P(v)$. 
Note that $\left(w_{n}^{-}\right)^{\bullet \mathrm{R}}=w_{n}$ and for $i \neq n,\left(\underline{w}_{i}^{-}\right)^{\bullet \mathrm{R}}=w_{i}^{-}$and $\left(w_{i}^{-}\right)^{\bullet \mathrm{R}}=\underline{w}_{i}^{-}$. That is, $\left(w_{I}^{-}\right)^{\bullet \mathrm{R}}=w_{[n-1] \backslash I}^{-}$, and thus the shape of $P\left(w_{I}^{-}\right)$is the 180 degree rotation of the shape of $P\left(w_{[n-1] \backslash I}^{-}\right)$. Therefore, the operation $\bullet \mathrm{R}$ establishes a bijection between the commutation classes $\left[w_{I}^{-}\right]$and $\left[w_{[n-1] \backslash I}^{-}\right]$. In particular, the commutation classes of $w_{0}=w_{[n-1]}^{-}$and $w_{0}^{\bullet \mathrm{R}}=w_{\emptyset}^{-}$have the same number of elements. For $n=5$, the shapes corresponding to the words in the classes of $w_{0}=w_{[n-1]}^{-}$and $w_{\emptyset}^{-}$are, respectively:
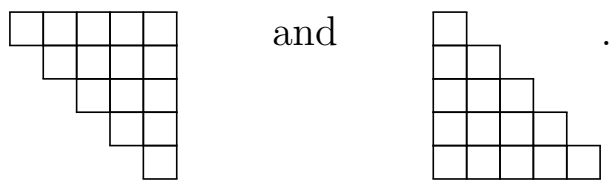

Note that $P\left(w_{0}\right)$ is a shifted standard Young tableau, i.e. a standard Young tableau of shifted shape given by the strict partition $(n, n-1, \ldots, 1)$. The bijection $P$ extends the bijection between reduced words in the class of $w_{0}$ and standard Young tableau of shifted shape given by $(n, n-1, \ldots, 1)$ constructed in [10]. A formula for the number of these standard Young tableau of shifted shape can be found in [11].

Proposition 43 (See [10]). The commutation classes of $w_{\emptyset}^{-}$and of $w_{[n-1]}^{-}$have

$$
\left(\begin{array}{l}
n \\
2
\end{array}\right) ! \prod_{i=0}^{n-2} \frac{i !}{(2 i+1) !}
$$

elements each.

Proof. It is proven in [10] that the number of reduced words in the commutation class of $w_{0}=w_{[n-1]}^{-}$is given by (6). This is also the number of elements in the commutation class of the word $w_{\emptyset}^{-}$, since $w_{0}^{\bullet R}=w_{\emptyset}^{-}$and $\bullet$ R establishes a bijection between the commutation classes $\left[w_{[n-1]}^{-}\right]$and $\left[w_{\emptyset}^{-}\right]$.

If $I \cup J$ is a partition of $[n-1]$, then we have $\left(w_{I}^{-}\right)^{\mathrm{R}}=w_{J}^{+}$, and the shape of the tableau of $\left(w_{I}^{-}\right)^{\mathrm{R}}$ is the reflection under the diagonal line $(i, j) \mapsto(j, i)$ of the tableau of $w_{I}^{-}$. Moreover, if the entry of position $(i, j)$ of $P\left(w_{I}^{-}\right)$is $a$, then the entry in position $(j, i)$ of $P\left(\left(w_{I}^{-}\right)^{\mathrm{R}}\right)$ is $n-a+1$. It follows that

$$
\left(w_{I}^{-}\right)^{\mathrm{R}}=w_{J}^{+} \sim w_{I^{\prime}}^{-},
$$

where $I^{\prime}=\{n-i: i \in I\}$. Therefore, the operation $\mathrm{R}$ establishes a bijection between the commutation classes of $\left[w_{I}^{-}\right]$and $\left[w_{I^{\prime}}^{-}\right]$, for each $I \subseteq[n-1]$. Since the operations $\bullet$ and $\mathrm{R}$ are involutions that commute with each other, it follows that the operation $\bullet$ establishes a bijection between the commutation classes of $\left[w_{I}^{-}\right]$and $\left[w_{[n-1] \backslash I^{\prime}}^{-}\right]$, for each $I \subseteq[n-1]$.

Lemma 44. Let $w_{I}^{\mathfrak{b}} \in \mathcal{T} \mathcal{S O}(n)$. Then $w_{I}^{\mathfrak{b}}$ is an alternating word if and only if $\mathfrak{b}$ has constant sign. 
Proof. When $\mathfrak{b}=(-)^{n-1}$ the word $w_{I}^{-}$is an element of the set $V \subseteq A R\left(\mathrm{w}_{\mathbf{0}}\right)$, and if $\mathfrak{b}=(+)^{n-1}$ then, as we have seen above, $w_{I}^{+} \sim w_{I^{\prime}}^{-}$, where $I^{\prime}=\{n-i: i \notin I\}$. Now, suppose $\mathfrak{b}$ is not a constant vector and let $i \in\{2, \ldots, n-1\}$ be the first index such that $\mathfrak{b}_{i} \neq \mathfrak{b}_{i-1}$. We assume $\mathfrak{b}_{i}=-$ (the other case is analogous), and consider a two sided ordered word $w_{I}^{\mathfrak{b}}$. Note that if $a \leqslant c<d<b$, then the word $t_{a, b}^{+} \cdot t_{c, d}^{-}$is not alternating, since $\left(t_{a, b}^{+} \cdot t_{c, d}^{-}\right)_{\mid\{d-1, d\}}=(d-1) d^{2}(d-1)$. If $i \notin I$, then $w_{I}^{\mathfrak{b}}$ has a factor $w_{n-j+1}^{\mathfrak{b}_{j}} \cdot w_{n-i+1}^{\mathfrak{b}_{i}}=t_{a, b}^{+} \cdot t_{c, d}^{-}$, for some $j<i$ and $a \leqslant c<d<b$. Similarly, if $i \in I$, then $w_{I}^{\mathfrak{b}}$ has a factor $\underline{w}_{n-i+1}^{\mathfrak{b}_{i}} \cdot \underline{w}_{n-j+1}^{\mathfrak{b}_{j}}=t_{c, d}^{-} \cdot t_{a, b}^{+}$, for some $j<i$ and $a \leqslant c<d<b$, where we let $\underline{w}_{n-j+1}^{\mathfrak{b}_{j}}=w_{n}^{\mathfrak{b}_{1}}$ if $j=1$. It follows that $w_{I}^{\mathfrak{b}}$ is not an alternating word.

We are now ready to compute the number of commutation classes having an element of the set $\mathcal{T S O}(n)$ as a representative. Given $w_{I}^{\mathfrak{b}} \in \mathcal{T} \mathcal{S O}(n)$ and $i \in[n]$, define the integers

$$
x_{i}:=1+\#\left\{j<i: \mathfrak{b}_{j}=+\right\} \quad \text { and } \quad y_{i}:=n+1-\#\left\{j<i: \mathfrak{b}_{j}=-\right\} .
$$

Note that the permutation associated with $w_{n+1-i}^{\mathfrak{b}_{i}}$ sends $x_{i}$ to $y_{i}$ if $\mathfrak{b}_{i}=+$, and sends $y_{i}$ to $x_{i}$ if $\mathfrak{b}_{i}=-$.

Lemma 45. Let $w_{I}^{\mathfrak{b}} \in \mathcal{T} \mathcal{S O}(n)$. For $i \in[n]$, define the sets

$$
\Delta^{i}=\left\{\begin{array}{ll}
\left\{\left(x_{i}, z\right): x_{i}<z \leqslant y_{i}\right\}, & \text { if } \mathfrak{b}_{i}=+ \\
\left\{\left(z, y_{i}\right): x_{i} \leqslant z<y_{i}\right\}, & \text { if } \mathfrak{b}_{i}=-
\end{array} \quad \text { and } \quad \Delta_{I}^{i}=\bigcup_{\substack{j>i \\
n+1-j \in I}} \Delta^{j}\right.
$$

Then, we have

$$
T\left(w_{I}^{\mathfrak{b}}, x_{i} z y_{i}\right)=\left\{\begin{array}{ll}
+1, & \text { if }\left(\mathfrak{b}_{i}=+ \text { and }\left(z, y_{i}\right) \notin \Delta_{I}^{i}\right) \text { or }\left(\mathfrak{b}_{i}=- \text { and }\left(x_{i}, z\right) \in \Delta_{I}^{i}\right) \\
-1, & \text { if }\left(\mathfrak{b}_{i}=- \text { and }\left(x_{i}, z\right) \notin \Delta_{I}^{i}\right) \text { or }\left(\mathfrak{b}_{i}=+ \text { and }\left(z, y_{i}\right) \in \Delta_{I}^{i}\right)
\end{array} .\right.
$$

Proof. Let $i \in[n]$ and assume $\mathfrak{b}_{i}=+$ (the other case is analogous). The set $\Delta_{I}^{i}$ stores all pairs of integers $(a, b)$ with $a<b$ such that $\mathrm{w}(a)>\mathrm{w}(b)$ for $\mathrm{w}$ the permutation associated with one of the factors $\underline{w}_{n+1-j}^{\mathfrak{b}_{j}}$, with $j>i$. Then the permutation associated with $w_{I}^{\mathfrak{b}}$ sends $x_{i}$ to $y_{i}$. If the pair $\left(z, y_{i}\right)$ was not inverted by now (that is, $\left.\left(x_{i}, z\right) \in \Delta_{I}^{i}\right)$, then the pair $\left(x_{i}, z\right)$ is inverted before the pair $\left(z, y_{i}\right)$ and, consequently, $T\left(w_{I}^{\mathfrak{b}}, x_{i} z y_{i}\right)=+1$.

Proposition 46. The elements of the set $\mathcal{T S O}(n)$ belong to $3 \times 4^{n-2}-2^{n-1}$ distinct commutation classes.

Proof. By Theorem 34, the number of commutation classes in the set of all alternating words $A R\left(\mathrm{w}_{\mathbf{0}}\right)$ is $2^{n-1}$, and by Lemma 44, each one of these classes has exactly two words from $\mathcal{T S O}(n)$. Consequently, using Lemma 23 we conclude that there are at most $3 \times 4^{n-2}-2^{n-1}$ distinct commutation classes in the set $\mathcal{T} \mathcal{S O}(n)$.

If two $\mathcal{T} \mathcal{S O}(n)$ words $w_{I}^{\mathfrak{b}}$ and $w_{I^{\prime}}^{\mathfrak{b}^{\prime}}$ are different and have the same $T$-value for all triples of integers in $[n+1]$, then by Lemma $45, \mathfrak{b}$ and $\mathfrak{b}^{\prime}$ have opposite constant signs and $I^{\prime}=[n-1] \backslash I$. By Lemma 44, both words are alternating words. 
In the next result, we prove that the cardinality of the commutation class of an alternating word $v \in V$ is a local maximum; that is, the cardinality of $[v]$ is the largest among all classes connected to it by a single long relation.

Proposition 47. The class $[v]$ is a local maximum, for any alternating reduced word $v \in V$.

Proof. Let $I=\left\{i_{1}, \ldots, i_{k}\right\}$ and $w_{I}^{-} \in V$, with $\left\{j_{1}, \ldots, j_{\ell}\right\}=[n-1] \backslash I$ and $j_{1}<\cdots<j_{\ell}$. A reduced word $w \notin\left[w_{I}^{-}\right]$is connected to some word $v \in\left[w_{I}^{-}\right]$only by a long relation $121 \sim 212$ or $n(n-1) n \underset{\mathrm{L}}{\sim}(n-1) n(n-1)$, since between two consecutive letters $i$ of $w_{I}^{-}$, with $i \neq 1, n-1$, there is always a letter $i-1$ or a letter $i+1$, by the definition of an alternating word. A long relation $121 \sim 212$ is obtained by using short relations in $w_{I}^{-}$between the letters 1 and 21 of two subwords $\underline{w}_{i_{q}}^{-}$and $\underline{w}_{i_{q+1}}^{-}$, respectively (assuming $\underline{w}_{i_{q+1}}^{-}=w_{n}^{-}$if $\left.q=k\right)$, in order to form a factor 121. Similarly, a long relation $n(n-1) n \sim$ $(n-1) n(n-1)$ is obtained by using short relations in $w_{I}^{-}$between the letters $n(n-1)$ and $n$ of two subwords $w_{j_{p+1}}^{-}$and $w_{j_{p}}^{-}$, respectively (assuming $w_{j_{p+1}}^{-}=w_{n}^{-}$if $p=\ell$ ), in order to form a factor $n(n-1) n$.

Let $v=t_{1} t t_{2} \sim w_{I}^{-}$be a word in the commutation class of $w_{I}^{-}$with a factor $t=121$, and let $w=t_{1} \bar{t} t_{2} \sim v$, with $\bar{t}=212$. Any sequence of short relation on the factor $t_{1}$ or $t_{2}$ of $w$ can be replicated in $v$. Moreover, a sequence of short relations which uses one letter 2 in $\bar{t}$ can be replicated with the corresponding letter 1 of $t$. This defines an injection $f$ from the set $[w]$ into $[v]$. Note also that the sequence of short relations in $v$ that sends the letter 1 of $t$ to the opposite side of the closest letter 3 is not in $f([w])$, showing that the cardinality of $[w]$ is strictly less than that of $\left[w_{I}^{-}\right]$. An analogous argument shows that if $w$ is connected with $v \sim w_{I}^{-}$by a long relation $n(n-1) n \sim(n-1) n(n-1)$, then the cardinality of $[w]$ is again strictly less than the cardinality of $\left[w_{I}^{-}\right]$. Therefore, we can conclude that $\left[w_{I}^{-}\right]$is a local maximum.

Among the cardinalities of all classes in $R\left(\mathrm{w}_{\mathbf{0}}\right)$, the four atoms we described in Theorem 27 are the classes with fewest elements, and the last result shows that each alternating class $\left[w_{I}^{-}\right]$, with $I \subset[n-1]$ is a local maximum. Computational evidence leads to the following conjecture.

Conjecture. The alternating classes of $w_{I}^{-}$and $\left(w_{I}^{-}\right)^{\bullet \mathrm{R}}=w_{J}^{-}$are the ones with maximum cardinality amongst all classes of $R\left(\mathrm{w}_{\mathbf{0}}\right)$, where $I$ and $J$ are, respectively, the sets of all odd numbers and all even numbers in $[n-1]$.

For instance, when $n=6$ we have $w_{I}^{-}=1 \cdot 321 \cdot 54321 \cdot 654321 \cdot 6543 \cdot 65$ and $w_{J}^{-}=21 \cdot 4321 \cdot 654321 \cdot 65432 \cdot 654 \cdot 6$. Moreover, when restricted to the alternating classes in $A R\left(\mathbf{w}_{\mathbf{0}}\right)$, we conjecture that the classes $w_{0}=w_{[n-1]}^{-}$and $w_{\emptyset}^{-}$are the ones having fewest elements, so that $\left|\left[w_{0}\right]\right| \leqslant|[w]| \leqslant\left|w_{I}^{-}\right|$for any $w \in A R\left(\mathbf{w}_{\mathbf{0}}\right)$. 


\section{Acknowledgements}

We thank the anonymous reviewer for a careful reading of the manuscript and many insightful comments and suggestions.

\section{References}

[1] R. Adin and Y. Roichman (2014). Enumeration of Standard Young Tableaux. arXiv: 1408.4497.

[2] S.H. Assaf (2019). An inversion statistic for reduced words. Advances in Applied Mathematics, 107, 1-21.

[3] N. Bergeron, C. Ceballos, and J. Labbé (2015). Fan Realizations of Type A Subword Complexes and Multi-associahedra of Rank 3. Discrete \& Computational Geometry, 54, 195-231.

[4] A. Björner and F. Brenti, Combinatorics of Coxeter groups, Graduate Texts in Mathematics 231, Springer, New York 2005.

[5] G. Chartrand, L. Lesniak and P. Zhang, Graphs \& Digraphs (5th ed.), CRC Press, 2010.

[6] H. Denoncourt, D. Ernst, and D. Story (2016). On the number of commutation classes of the longest element in the symmetric group. Open Problems in Mathematics, Volume 4.

[7] S. Elnitsky (1997). Rhombic tilings of polygons and classes of reduced words in Coxeter groups, Journal of Combinatorial Theory, Series A, 77, 193-221.

[8] M. Geck and G. Pfeiffer, Characters of finite Coxeter groups and Iwahori-Hecke algebras. London Mathematical Society Monographs, 2000.

[9] V. Reiner and Y. Roichman (2013). Diameter of graphs of reduced words and galleries. Transactions of the American Mathematical Society, 365(5), 2779-2802.

[10] A. Schilling, N.M. Thiéry, G. White and N. Williams (2017). Braid moves in commutation classes of the symmetric group. European Journal of Combinatorics, 62, $15-34$.

[11] B. Sagan (1980). On Selecting a Random Shifted Young Tableau. Journal of Algorithms, 1, 213-234.

[12] R.P. Stanley (1984). On the number of reduced decompositions of elements of Coxeter groups. European Journal of Combinatorics, 5, 359-372.

[13] B.E. Tenner (2006). Reduced decompositions and permutation patterns. Journal of Algebraic Combinatorics, 24, 263-284. 